\title{
The elementary computable functions over the real numbers: Applying two new techniques
}

\author{
Manuel L. Campagnolo \\ D.M./I.S.A., Lisbon University of Technology and SQIG/IT \\ mlc@math.isa.utl.pt \\ Kerry Ojakian \\ SQIG - IT and IST, Portugal \\ ojakian@math.ist.utl.pt
}

July 6, 2007

\begin{abstract}
The basic motivation behind this work is to tie together various computational complexity classes, whether over different domains such as the naturals or the reals, or whether defined in different manners, via function algebras (Real Recursive Functions) or via Turing Machines (Computable Analysis). We provide general tools for investigating these issues, using two techniques we call approximation and lifting. We use these methods to obtain two main theorems. First we provide an alternative proof of the result from Campagnolo, Moore and Costa [3], which precisely relates the Kalmar elementary computable functions to a function algebra over the reals. Secondly, we build on that result to extend a result of Bournez and Hainry [1], which provided a function algebra for the $\mathcal{C}^{2}$ real elementary computable functions; our result does not require the restriction to $\mathcal{C}^{2}$ functions. In addition to the extension, we provide an alternative approach to the proof. Their proof involves simulating the operation of a Turing Machine using a function algebra. We avoid this simulation, using a technique we call lifting, which allows us to lift the classic result regarding the elementary computable functions to a result on the reals. The two new techniques bring a different perspective to these problems, and furthermore appear more easily applicable to other problems of this sort.
\end{abstract}

\section{Introduction}

This paper improves, corrects, and extends the ideas in our earlier paper [4]. We will study classes of functions with respect to their computational complexity, showing connections between different models of computation. The classic case is concerned with classes of functions whose domain and range are the natural numbers, $\mathbb{N}$, where the functions are defined via Turing Machines or an equivalent model of computation. In this case, there is one agreed upon concept of computation and computational complexity with different models yielding the same set of functions. More recent work has extended computational complexity to classes of functions over the real numbers, $\mathbb{R}$, but in this case there is not one agreed upon notion of computation. We will concentrate on two models of computation over the reals, "The Real Recursive Functions" and "Computable Analysis." The former originated with Moore [8] (with some problems corrected by Costa and 
Mycka in [9] and [10]) and the latter with Grzegorczyk [6]. In Computable Analysis, Turing Machines are used to characterize various classes of functions over the reals, with the idea being that a real function is computable by a Turing Machine if it can be approximated to the appropriate level of precision from approximations to the input of the function. In the case of Real Recursive Functions, classes of functions are defined using function algebras in which the discrete operations of recursion are replaced by operations which find solutions to differential equations. Our goal is to study connections between these three different kinds of function classes, the classic ones over the naturals, the ones arising from Computable Analysis, and the ones arising from Real Recursive Functions.

There have been a number of results tieing together these three different models of computation. Campagnolo, Moore and Costa [3] describe a class of real functions (they call $\mathcal{L}$ ), defined via a function algebra in which the principle operation is to obtain the solution of a system of linear differential equations. They show that the "discrete part" (definition 4.1) of $\mathcal{L}$ is exactly the usual Kalmar elementary computable functions on the naturals. Building on this, Bournez and Hainry [1] show that $\mathcal{L}$ extended by a certain limit operation is the class of $\mathcal{C}^{2}$ elementary computable functions on $\mathbb{R}$. In section 4 , we provide an alternative proof of the result of [3]. In sections 5 and 6 we extend the result from [1]; in particular, we show that $\mathcal{L}$ augmented by any of a number of limit operations yields exactly the elementary computable functions on $\mathbb{R}$, without a restriction to the $\mathcal{C}^{2}$ functions.

The novelty we bring to these problems are two new techniques, which we call "approximation" and "lifting." The first technique is used throughout the paper. The basic idea of this technique is to define a general kind of approximation relation that can hold between two classes of functions $\mathcal{A}$ and $\mathcal{B}$. Roughly speaking we will say that $\mathcal{B}$ approximates $\mathcal{A}$, if for any required precision, any function of $\mathcal{A}$ can be approximated to that precision with a function from $\mathcal{B}$; this will be written, roughly, as $\mathcal{A} \preceq \mathcal{B}$. Our approach to both the theorems of this paper is to first show that two classes of functions approximate each other, and then derive the desired equality from the sufficiently close approximation. Under the right conditions, the approximation relation will in fact be a transitive relation, thus allowing us to break down an approximation into a series of smaller and more natural tasks. Using approximation, our alternative proof of the result from [3] follows more closely the inductive definitions of the function algebras in question (discussed in more detail in section 4).

The second technique, lifting, is the main tool used in sections 5 and 6 to provide a function algebra for the elementary computable functions on $\mathbb{R}$. The lifting process begins with a known complexity result on $\mathbb{N}$, such as the fact that the elementary time functions defined via Turing Machines are exactly the functions given by a particular function algebra. The lifting can be seen as a two step process (discussed in detail in section 5). First we lift the result on $\mathbb{N}$ to an analogous result on the rationals, $\mathbb{Q}$, where the model essentially treats the rationals as pairs of natural numbers. The second step (the most involved one) is to lift this to a result on $\mathbb{R}$. In the work of [1], the proof involves coming up with a new Turing Machine simulation of the class of elementary computable functions on $\mathbb{R}$; we manage to avoid using a new Turing Machine simulation, by reusing a classic result on $\mathbb{N}$ (which of course involves a Turing Machine simulation) and lifting this to $\mathbb{R}$.

We would like to claim that the advantages of these techniques are twofold. First, they provide a different approach to some of these problems, which seems to facilitate working on these problems, especially when dealing with function algebras. Second, the techniques appear to be more amenable to generalization and wider application than some of the earlier approaches. We claim this based on other work in progress, and based on the character of the development. While some of lemmas 
are general, not specific to the elementary computable functions, a number of others could (with some adaptation) be stated in a more general way. The wider vision for this approach is a collection of general tools with broad application. We present the beginning of such a development.

The paper is organized as follows. In section 2 we define the main concepts that are going to be used throughout the paper. In section 3 we discuss how those concepts can be used with function algebras. In section 4 we consider the elementary computable functions and prove our first main result. In section 5 we establish links with computable analysis and present our second main result. To improve readability we leave the more technical discussion to section 6 . Finally, we point out some directions for further research.

\section{Approximation}

To develop formally the definition of approximation we will need to be able to talk about functions and their arguments in a precise way. If a function $f\left(x_{1}, \ldots, x_{k}\right)$ is defined on exactly $X^{k}$ and takes values in $X$, we say it is an $X$-function, or equivalently, that it has universe $X$; we do not consider vector valued functions. We always assume the universe is a subset of $\mathbb{R}$, and in fact the only particular cases we consider in this paper will be $\mathbb{N}, \mathbb{Q}$, and $\mathbb{R}$. To refer to function arguments precisely we will sometimes need to speak more formally about the variables of a function. We use lower case letters to refer to variables and put a bar over the letter, such as $\bar{x}$, to refer to a finite list of variables (if not stated or implied by context, a list may be empty). When we write lists of variables separated by comas, within a function, such as $f(\bar{x}, \bar{y})$, we intend that the variables are all distinct. If we have lists of variables or numbers $\bar{a}$ and $\bar{b}$, the same lengths as $\bar{x}$ and $\bar{y}$, respectively, then by $f(\bar{a}, \bar{b})$ we mean to substitute $\bar{a}, \bar{b}$ for $\bar{x}, \bar{y}$ in order. The key point is that given two functions, the notion of variables allows us to associate the arguments of two different functions in any way we wish. Any classes of functions we work with will be sufficiently strong that we can freely manipulate variables in typical ways. For the approximation relation we will use functions to translate between different universes.

Definition 2.1 Suppose $A, B \subseteq \mathbb{R}$. An interpretation from $A$ to $B$ is a (possibly partial) injection from $A$ to $B$.

Notice that an interpretation is a function of one argument; if $\omega$ is an interpretation and we write $\omega\left(\left(a_{1}, \ldots, a_{k}\right)\right)$, we mean $\left(\omega\left(a_{1}\right), \ldots, \omega\left(a_{k}\right)\right)$. For $a \in A$, we call $\omega(a) \in B$ the code of $a$, and conversely, for $b \in B$, we say that $b$ codes $\omega^{-1}(b) \in A$. We will now define the approximation notion between classes of functions, building on the notion of approximation between two single functions. Some examples follow the definitions.

Definition 2.2 Suppose $A, B \subseteq \mathbb{R}$. Suppose $f(\bar{x})$ is a function, on universe $A$, and $h(\bar{x}, \bar{y})$ is a function on universe $B$. Suppose $\varepsilon(\bar{x}, \bar{y})$ is a function on universe $\mathbb{R}$. Suppose $\omega: A \rightarrow B$ is an interpretation. By

$$
f \preceq^{\varepsilon,[\omega]} h,
$$

we mean that for all $\bar{x}, \bar{y} \in \operatorname{Domain}(\omega), h(\omega(\bar{x}), \omega(\bar{y}))$ is in the domain of $\omega^{-1}$, and the following holds:

$$
\left|f(\bar{x})-\omega^{-1} \circ h(\omega(\bar{x}), \omega(\bar{y}))\right| \leq \varepsilon(\bar{x}, \bar{y}) .
$$


Definition 2.3 Let $\mathcal{A}, \mathcal{B}$, and $\mathcal{E}$ be classes of functions with universes $A, B$, and $\mathbb{R}$, respectively, such that $A, B \subseteq \mathbb{R}$. Suppose $\omega: A \rightarrow B$ is an interpretation.

- We write

$$
\mathcal{A} \preceq{ }_{-}^{\mathcal{E},[\omega]} \mathcal{B}
$$

to mean that for any $f(\bar{x}) \in \mathcal{A}$ and $\varepsilon(\bar{x}) \in \mathcal{E}$, there is $h(\bar{x}) \in \mathcal{B}$, such that $f \preceq^{\varepsilon,[\omega]} h$.

- We write

$$
\mathcal{A} \preceq_{+}^{\mathcal{E},[\omega]} \mathcal{B}
$$

to mean that for any $f(\bar{x}) \in \mathcal{A}$ and $\varepsilon(\bar{x}, \bar{y}) \in \mathcal{E}$, there is $h(\bar{x}, \bar{y}) \in \mathcal{B}$, such that $f \preceq^{\varepsilon,[\omega]} h$.

Note that in the definition of approximation, the approximation condition is required to hold for any precision $\varepsilon \in \mathcal{E}$; in many applications it would suffice to just have one $\varepsilon \in \mathcal{E}$, yet it appears easier to inductively prove approximations for the stronger notion we use. At the end of this section we comment more on this particular approximation compared to other possibilities. Recalling that lists of variables may be empty, the approximation with the "+" is stronger than the one with the "-". Consider some useful conventions regarding the approximation notation.

Remark 2.4 Suppose $\mathcal{A}$ and $\mathcal{B}$ are sets of functions on universes $A$ and $B$ respectively. Consider $\mathcal{A} \preceq_{+/-}^{\mathcal{E},[\omega]} \mathcal{B}$.

- If $\omega$ is missing we mean for $\omega=i d_{A \cap B}$ (the identity function on $A \cap B$ ).

- When it is clear that something is an interpretation, we may omit the square brackets.

- If $\mathcal{E}$ is missing, we assume $\mathcal{E}=$ Zero, where Zero is the set of all $\mathbb{R}$-functions that have value 0 everywhere (i.e. the "approximation" must have no error).

- If we leave out "+" and "-", we mean "-".

- If we use "+/-" in a statement we mean that it holds for "+" substituted everywhere for "+/-", or for "-" substituted everywhere for "+/-".

- For classes of functions $\mathcal{A}_{i}$ with universe $A$, we write $\mathcal{A}_{1}, \ldots, \mathcal{A}_{k} \preceq_{+/-} \mathcal{E}$, $[\omega]$ Bo mean $\mathcal{A}_{1} \preceq_{+/-}^{\mathcal{E},[\omega]} \mathcal{B}, \ldots, \mathcal{A}_{k} \preceq_{+/-}^{\mathcal{E},[\omega]} \mathcal{B}$.

For a simple example consider the expression $\mathcal{A} \preceq \mathcal{B}$, where $\mathcal{A}$ and $\mathcal{B}$ are classes of functions on universes $A$ and $B$, respectively. By convention it abbreviates $\mathcal{A} \preceq_{-}^{\left.\text {Zero, [id }{ }_{A \cap B}\right]} \mathcal{B}$. If $A=B$, then it just means $\mathcal{A} \subseteq \mathcal{B}$. If $A \subseteq B$ then the expression means any function of $\mathcal{A}$ can be extended to a function in $\mathcal{B}$ so that their values coincide on $A$. If $B \subseteq A$ then the expression means that $\mathcal{B}$ contains the restriction to $B$ of any function from $\mathcal{A}$.

We now introduce the interpretation and related functions that we will work with throughout this paper.

\section{Definition 2.5}


- Let pair $(a, b)=(1 / 2)(a+b+1)(a+b)+a($ a bijection from $\mathbb{N} \times \mathbb{N}$ to $\mathbb{N})$.

- When we say that a rational is presented in lowest terms we mean that it is given to us as $(-1)^{s}(a / b)$, where either $a=b=s=0$, or $a, b \in \mathbb{N}, s \in\{0,1\}$, with $a, b>0$ and $a$ and $b$ relatively prime.

- We define an interpretation $\lambda: \mathbb{Q} \rightarrow \mathbb{N}$. For any rational $(-1)^{s}$ a/b presented in lowest terms, let $\lambda\left((-1)^{s} a / b\right)=2$ pair $(a, b)+s$.

Definition 2.6 We define some functions on universe $\mathbb{N}$.

- $\operatorname{parity}(n)= \begin{cases}0, & \text { if } n \text { even; } \\ 1, & \text { if } n \text { odd }\end{cases}$

- $\operatorname{gcd}(a, b)=$ the greatest common divisor of $a$ and $b$ (we let $\operatorname{gcd}(a, b)=0$ if $a$ or $b$ is zero)

- top and bot are the unique functions from $\mathbb{N}$ to $\mathbb{N}$ such that for any rational $(-1)^{s}$ a/b presented in lowest terms, $\operatorname{top}\left(\lambda\left((-1)^{s} a / b\right)\right)=a$, and bot $\left(\lambda\left((-1)^{s} a / b\right)\right)=b$.

- $\operatorname{code}(a, b, s)=2 \operatorname{pair}\left(\frac{a}{\operatorname{gcd}(a, b)}, \frac{b}{\operatorname{gcd}(a, b)}\right)+s=\lambda\left((-1)^{s} a / b\right)$, where we take " $x / 0$ " to be 0 (this function is motivated in the following example).

We will now work out a more involved example which we will in fact use later (in lemma 4.11).

Example 2.7 Consider the function mult $(x, y)=x y$, on $\mathbb{Q}$. Suppose we want a function mult $(n, m)$ on $\mathbb{N}$, such that it interprets mult (via $\lambda$ ), i.e. mult $\preceq^{\lambda}$ mult ${ }^{*}$, which by our convention means mult $\preceq-{ }_{-}^{\text {Zero, }[\lambda]}$ mult* and, in this case, amounts to the property:

$$
\lambda(\operatorname{mult}(x, y))=\operatorname{mult} t^{*}(\lambda(x), \lambda(y)), \text { for } x, y \in \mathbb{Q} .
$$

Given 2 rationals presented in lowest terms as $(-1)^{k}(p / q)$, and $(-1)^{c}(a / b)$, their product is arrived at by multiplying the tops of the fractions together, dividing by the product of the bottoms, and taking account of the sign, to attain $(-1)^{k+c} p a / q b$, where the presentation may no longer be in lowest terms. To define mult* we carry out the same kind of procedure, but on the natural numbers $n$ and $m$ which code rationals. Thus the top should be top $(n)$ top $(m)$, the bottom should be $\operatorname{bot}(n) \operatorname{bot}(m)$, and the sign should be $s(n, m)=\operatorname{delta}(n) \operatorname{delta}(m) \operatorname{parity}(\operatorname{parity}(n)+\operatorname{parity}(m))$, where delta $(x)=0$ if $x=0$ and 1 otherwise. Not only does mult* receive codes ( $n$ and $m$ ) as input, but it must output a code, thus the output should be $\lambda\left((-1)^{s(n, m)} \frac{\operatorname{top}(n) \operatorname{top}(m)}{\operatorname{bot}(n) \operatorname{bot}(m)}\right)$, so we define $\operatorname{mult} t^{*}(n, m)=\operatorname{code}(\operatorname{top}(n) \operatorname{top}(m)$, bot $(n) \operatorname{bot}(m), s(n, m))$, which works by the property of code noted above.

Here and throughout the paper, we will use the important technical idea of linearizing a function defined on $\mathbb{Z}$, that is extending it to domain $\mathbb{R}$, by basically connecting the values on $\mathbb{Z}$ by straight lines; by $\lfloor x\rfloor$ we mean the greatest integer less than or equal to $x$, and by $\lceil x\rceil$ we mean the smallest integer greater than or equal to $x$. 
Definition 2.8 Suppose the domain of $f\left(x_{1}, \ldots, x_{k}\right)$ contains $\mathbb{Z}$ (its codomain may be $\mathbb{R}$ ), and $r \leq k$. We define an operation which outputs an $\mathbb{R}$-function $h\left(x_{1}, \ldots, x_{k}\right)=\operatorname{Lin}_{\mathbb{R}}\left(f ; x_{1}, \ldots, x_{r}\right)=$

$$
\begin{aligned}
& f\left(\left\lfloor x_{1}\right\rfloor, \ldots,\left\lfloor x_{r}\right\rfloor, x_{r+1}, \ldots, x_{k}\right)\left(\left\lfloor x_{1}\right\rfloor+1-x_{1}\right) \ldots\left(\left\lfloor x_{r}\right\rfloor+1-x_{r}\right) \\
& +f\left(\left\lceil x_{1}\right\rceil,\left\lfloor x_{2}\right\rfloor, \ldots,\left\lfloor x_{r}\right\rfloor, x_{r+1}, \ldots, x_{k}\right)\left(x_{1}-\left\lfloor x_{1}\right\rfloor\right)\left(\left\lfloor x_{2}\right\rfloor+1-x_{2}\right) \ldots\left(\left\lfloor x_{r}\right\rfloor+1-x_{r}\right) \\
& \quad \vdots \\
& +f\left(\left\lceil x_{1}\right\rceil, \ldots,\left\lceil x_{r}\right\rceil, x_{r+1}, \ldots, x_{k}\right)\left(x_{1}-\left\lfloor x_{1}\right\rfloor\right) \ldots\left(x_{r}-\left\lfloor x_{r}\right\rfloor\right),
\end{aligned}
$$

where the intention is to range over all $2^{r}$ combinations of $\lfloor\cdot\rfloor$ and $\lceil\cdot\rceil$ applied to the $x_{i}$; corresponding to whether $\lfloor x\rfloor$ or $\lceil x\rceil$ is applied, we multiply $f$ by $(\lfloor x\rfloor+1-x)$ or $(x-\lfloor x\rfloor)$, respectively. We write $\hat{f}$ to indicate the full linearization, $\operatorname{Lin}_{\mathbb{R}}\left(f ; x_{1}, \ldots, x_{k}\right)$.

We write $\operatorname{Lin}_{\mathbb{Q}}$ to indicate exactly the same operation, except that the input function must have codomain $\mathbb{Q}$, and the function, $h$, that is output is only defined on $\mathbb{Q}$.

Note that the values of $f$ off of $\mathbb{Z}$ have no effect on $\hat{f}$. When we write just Lin it will be clear from context whether we mean to linearize with respect to $\mathbb{R}$ (i.e. use $\operatorname{Lin}_{\mathbb{R}}$ ), linearize with respect to $\mathbb{Q}$ (i.e. use $\operatorname{Lin}_{\mathbb{Q}}$ ), or refer to both simultaneously.

Proposition 2.9 Suppose $f\left(x_{1}, \ldots, x_{k}\right)$ is a function with a domain containing $\mathbb{Z}$, and we consider linearization with respect to $\mathbb{R}$ or $\mathbb{Q}$.

- For $\bar{x} \in \mathbb{Z}, \hat{f}(\bar{x})=f(\bar{x})$.

- $\hat{f}$ is continuous.

- For any $\bar{x}$ (in $\mathbb{R}$ or $\mathbb{Q}$ depending on which case we are considering), let

$$
\mathcal{X}(\bar{x})=\left\{f\left(\left\lfloor x_{1}\right\rfloor, \ldots,\left\lfloor x_{k}\right\rfloor\right), \ldots, f\left(\left\lceil x_{1}\right\rceil, \ldots,\left\lceil x_{k}\right\rceil\right)\right\},
$$

where we range over all $2^{k}$ combinations of $\lfloor\cdot\rfloor$ and $\lceil\cdot\rceil$. The following holds:

$$
\min (\mathcal{X}(\bar{x})) \leq \hat{f}(\bar{x}) \leq \max (\mathcal{X}(\bar{x}))
$$

We now come to some definitions that for the purpose of this paper we could avoid. However, they facilitate some discussion and are are important for our goal of developing generally applicable techniques. We will define the concepts of "bounding class" and "error class." Intuitively, a class of functions is a bounding class if it can be used to measure the growth rate of some other class of functions. A class of functions is an error class, if it can be used to measure the error when one class of functions approximates another.

Definition 2.10 Suppose $f(y, \bar{x})$ is a function on universe A. We say it converges uniformly to infinity in $\boldsymbol{y}$ if for every $n>0$ there is $m_{0}>0$ such that for any $m, \bar{x} \in A, m \geq m_{0}$, we have $f(m, \bar{x}) \geq n$.

Definition 2.11 For $f: \mathbb{N} \rightarrow \mathbb{N}$, let $\tilde{f}: \mathbb{Z} \rightarrow \mathbb{N}$ be defined by $\tilde{f}(a)= \begin{cases}f(a), & \text { if } a \geq 0 ; \\ f(-a), & \text { if } a<0 .\end{cases}$ 
Definition 2.12 Suppose $\mathcal{B}^{*}$ is a class of functions on universe $\mathbb{N}$, such that for any $f(\bar{x}), g \in \mathcal{B}^{*}$ :

1. $f>0$

2. $f$ is increasing in any variable.

3. $f$ converges uniformly to infinity in any of its variables.

4. For any variables $\bar{y}$ disjoint from $\bar{x}$, there is $f^{*}(\bar{x}, \bar{y}) \in \mathcal{B}^{*}$ such that $f(\bar{x}) \leq f^{*}(\bar{x}, \bar{y})$.

5. There are $h_{1}, h_{2} \in \mathcal{B}^{*}$ such that $f+g \leq h_{1}$ and $f \circ g \leq h_{2}$.

Then $\mathcal{B}=\left\{\hat{\tilde{f}} \mid f \in \mathcal{B}^{*}\right\}$ is a bounding class.

We will be interested in a bounding class $\mathcal{T} \mathcal{W}$ which contains functions that grow like towers of 2 , that is at the speed of the elementary functions.

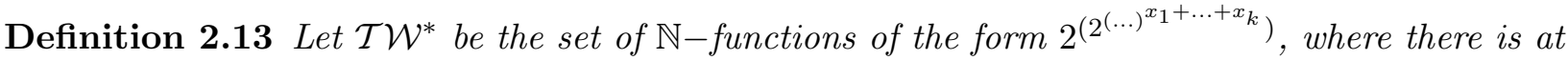
least one power of 2 and we can choose any number of variables.

Let $\mathcal{T} \mathcal{W}=\left\{\hat{\tilde{f}} \mid f \in \mathcal{T} \mathcal{W}^{*}\right\}$.

Proposition 2.14 $\mathcal{T W}$ is a bounding class.

There is some flexibility in how we could have defined the idea of a bounding class; though the use of linearization could be avoided, we use linearization in other places anyway and it makes some of the technical development easier. We will form error classes by taking the reciprocal of a bounding class, i.e. for a set of functions $\mathcal{F}, 1 / \mathcal{F}=\{1 / f \mid f \in \mathcal{F}\}$.

Definition 2.15 An error class is either the set of functions Zero or the set of functions $1 / \mathcal{B}$ where $\mathcal{B}$ is a bounding class.

Now we justify the approximation notation by showing it is a partial order under the right conditions, that is it satisfies transitivity; when we reference "transitivity" in this paper we mean some application of the following lemma.

Lemma 2.16 (Transitivity) Suppose $\mathcal{A}, \mathcal{B}$, and $\mathcal{C}$ are classes of functions on universes $A, B$, and $C$, respectively, $\mathcal{E}$ is an error class, and $\omega: B \rightarrow C$ is an interpretation.

1. If $\mathcal{A} \preceq_{+}^{\mathcal{E}} \mathcal{B} \preceq_{+}^{\mathcal{E}} \mathcal{C}$ then $\mathcal{A} \preceq_{+}^{\mathcal{E}, \text { id }_{A \cap B \cap C} \mathcal{C}}$

2. If $\operatorname{Domain}(\omega) \subseteq A$ and $\mathcal{A} \preceq_{+}^{\mathcal{E}} \mathcal{B} \preceq^{\omega} \mathcal{C}$ then $\mathcal{A} \preceq_{+}^{\mathcal{E}, \omega} \mathcal{C}$

3. If $\gamma: A \rightarrow B$ is an interpretation such that domain $(\omega) \subset$ range $(\gamma)$, and $\mathcal{A} \preceq^{\gamma} \mathcal{B} \preceq^{\omega} \mathcal{C}$ then $\mathcal{A} \preceq^{\omega \circ \gamma} \mathcal{C}$

\section{Proof}


1. Let $f(\bar{x}) \in \mathcal{A}, \alpha(\bar{x}, \bar{y}) \in \mathcal{E}$ and we need $h(\bar{x}, \bar{y}) \in \mathcal{C}$ such that $|f(\bar{x})-h(\bar{x}, \bar{y})| \leq$ $\alpha(\bar{x}, \bar{y})$ for $\bar{x}, \bar{y} \in A \cap B \cap C$. Since $\mathcal{E}$ is an error class there is $\alpha^{*}(\bar{x}, \bar{y}) \in \mathcal{E}$ such that $\alpha^{*}(\bar{x}, \bar{y}) \leq(1 / 2) \alpha(\bar{x}, \bar{y})$. Let $g(\bar{x}, \bar{y}) \in \mathcal{B}$ such that $|f(\bar{x})-g(\bar{x}, \bar{y})| \leq \alpha^{*}(\bar{x}, \bar{y})$ for all $\bar{x}, \bar{y} \in A \cap B$. Let $h(\bar{x}, \bar{y}) \in \mathcal{C}$ such that $|g(\bar{x}, \bar{y})-h(\bar{x}, \bar{y})| \leq \alpha^{*}(\bar{x}, \bar{y})$ for $\bar{x}, \bar{y} \in B \cap C$. Thus $|f(\bar{x})-h(\bar{x}, \bar{y})| \leq \alpha^{*}(\bar{x}, \bar{y})+\alpha^{*}(\bar{x}, \bar{y}) \leq \alpha(\bar{x}, \bar{y})$, as required. Note that we need "+" for the first approximation, but "+" or "-" works for the second one.

2. Let $f(\bar{x}) \in \mathcal{A}$ and $\alpha(\bar{x}, \bar{y}) \in \mathcal{E}$ and we need $h(\bar{x}, \bar{y}) \in \mathcal{C}$ such that $\mid f(\bar{x})-\omega^{-1}$ 。 $h(\omega(\bar{x}), \omega(\bar{y})) \mid \leq \alpha(\bar{x}, \bar{y})$ for all $\bar{x}, \bar{y} \in \operatorname{Domain}(\omega)$. Let $g(\bar{x}, \bar{y}) \in \mathcal{B}$ such that $\mid f(\bar{x})-$ $g(\bar{x}, \bar{y}) \mid \leq \alpha(\bar{x}, \bar{y})$ for all $\bar{x}, \bar{y} \in A \cap B$. Let $h(\bar{x}, \bar{y}) \in \mathcal{C}$ such that $\mid g(\bar{x}, \bar{y})-\omega^{-1} \circ$ $h(\omega(\bar{x}), \omega(\bar{y})) \mid \leq 0$ for all $\bar{x}, \bar{y} \in \operatorname{Domain}(\omega)$. Thus $\left|f(\bar{x})-\omega^{-1} \circ h(\omega(\bar{x}), \omega(\bar{y}))\right| \leq$ $\alpha(\bar{x}, \bar{y})$ for all $\bar{x}, \bar{y} \in A \cap B \cap \operatorname{Domain}(\omega)$, which is enough since $\operatorname{Domain}(\omega) \subseteq A, B$. Note that the condition $\operatorname{Domain}(\omega) \subseteq A$ also ensures that $\mathcal{A} \preceq_{+}^{\mathcal{E}, \omega} \mathcal{C}$ makes sense.

3. Similar to others.

A useful shorthand is the following "approximate equality."

Definition 2.17 We write $\mathcal{A} \approx_{+/-}^{\mathcal{E}} \mathcal{B}$ to mean that both $\mathcal{A} \preceq_{+/-}^{\mathcal{E}} \mathcal{B}$ and $\mathcal{B} \preceq_{+/-}^{\mathcal{E}} \mathcal{A}$ hold.

Note that with the definition of approximation (with its particular quantifiers) it is important to read the definition in the right order. We use $\mathcal{B} \succeq_{+/-}^{\mathcal{E}} \mathcal{A}$ as another way to write $\mathcal{A} \preceq_{+/-}^{\mathcal{E}} \mathcal{B}$. Another important kind of relationship between classes of functions will be that of one class dominating another.

Definition 2.18 Suppose $\mathcal{A}$ and $\mathcal{B}$ are classes of functions on universes $A$ and $B$, respectively. We write $\mathcal{A} \leq \mathcal{B}$ if for every function $f(\bar{x}) \in \mathcal{A}$ there is a function $h(\bar{x}) \in \mathcal{B}$ such that $|f(\bar{x})| \leq h(\bar{x})$ for all $\bar{x} \in A \cap B$.

Much of the paper will consist of showing claims like " $\mathcal{A} \preceq_{+}^{1 / \mathcal{T W}} \mathcal{B}$ ", for various classes of functions $\mathcal{A}$ and $\mathcal{B}$. As indicated earlier, such claims and the rest of the work of this paper would in fact work fine with other notions of approximation. Another possibility would be to define approximation so that for $f^{*}(\bar{x}, \bar{y})$ to approximate $f(\bar{x})$ we only require $\left|f(\bar{x})-f^{*}(\bar{x}, \bar{y})\right| \leq \alpha(\bar{y})$, for some error function $\alpha(\bar{y})$, which has no variables $\bar{x}$, but only "parameter" variables. Or going further in this direction, we could require $\alpha$ to be exactly the function $1 / y$ with $f^{*}$ only having one extra parameter variable $y$. For the purposes of this paper, the notions would work in the same manner because if, for example, $f(\bar{x}) \preceq^{1 / t} f^{*}(\bar{x}, t)$, then $f(\bar{x}) \preceq^{1 / \alpha(\bar{x}, t)} f^{*}(\bar{x}, \alpha(\bar{x}, t))$. Thus if the classes of functions we work with are strong enough we can start with a function which approximates another one and improve the approximation by composition. Without going into the details, each approach seems to have advantages and disadvantages. We choose the particular definition we use since it both appears generalizable and facilitates some of the technical development.

\section{Function Algebras}

We will use function algebras to define most of our classes of functions. They are defined by giving some basic functions and closing the class under operations on functions. 
Definition 3.1 Suppose $\mathcal{A}$ is a class of functions on universe $A \subseteq \mathbb{R}$. An operation with universe $(\mathcal{A}, A)$ is a function which takes as input some functions from $\mathcal{A}$, and outputs a single function from $\mathcal{A}$. If $\mathcal{A}$ is all the functions with universe $A$, we say the operation has universe $A$.

Note that we require the universe of an operation to consist of functions which all have some fixed universe $A$; this $A$ may not always be referred to explicitly. As an example, we could define the typical operation (with universe $\mathbb{N}$ ) of bounded sum, denoted by $\sum$. The operation $\sum$ takes a function $f(y, \bar{x})$ as input and returns $g(z, \bar{x})=\sum_{y=0}^{z} f(y, \bar{x})$. Note that technically the operation should have some way of knowing which variable to carry out the summation upon, but we will ignore this minor issue here and for other operations.

Definition 3.2 Suppose $\mathcal{B}$ is a set of functions (called basic functions), and $\mathcal{O}$ is a set of operations. Then $F A[\mathcal{B} ; \mathcal{O}]$ is called a function algebra, and it denotes the smallest set of functions containing $\mathcal{B}$ and closed under the operations in $\mathcal{O}$. For ease of readability, we often list the elements of $\mathcal{B}$ or $\mathcal{O}$ simply as a list separated by commas.

An example of a function algebra we will use is the elementary computable functions defined via bounded sums and bound products. Let $\prod$ be the operation (called bounded products) on universe $\mathbb{N}$ which takes a function $f(y, \bar{x})$ and returns $g(z, \bar{x})=\prod_{y=0}^{z} f(y, \bar{x})$. Let comp be the operation which takes some functions (with any universe) and composes them.

Definition 3.3 Let basic $_{\mathbb{N}}$ be the following functions with universe $\mathbb{N}:+,-, P, 0,1$, where $P$ is the set of all projection functions on $\mathbb{N}$ and - is the usual cut-off subtraction, defined by

$$
x-y=\left\{\begin{array}{ll}
x-y & \text { if } x \geq y \\
0 & \text { otherwise }
\end{array} .\right.
$$

Thus $\mathrm{FA}\left[\right.$ basic $_{\mathbb{N}} ;$ comp, $\left.\sum, \Pi\right]$ is the elementary computable functions.

Definition 3.4 Let the function algebra $F A\left[\right.$ basic $_{\mathbb{N}} ;$ comp, $\left.\sum, \prod\right]$ be abbreviated by $F A_{\mathbb{N}}$.

Notice that for a function algebra, there can be 2 distinct ways to construct the same function. This highlights the syntactic side of a function algebra, which will become an issue in section 6 .

Definition 3.5 Given a function algebra $\mathcal{F}$, and $f \in \mathcal{F}$, by a construction tree of $f$ we mean a tree which describes a construction of $f$ in the function algebra. The leaves of this tree are labeled by various basic functions in the algebra, and internal nodes are labeled by operations in the algebra. Thus, we can think of the tree as specifying how to build a function, starting with the leaves and moving up the tree, so each node specifies a function in the algebra. For the tree to be associated to $f$, means that $f$ is the function associated with the root of the tree.

To compare function algebras it will be useful to talk about a class of functions, $\mathcal{B}$, approximating an operation; intuitively this means that if any functions are approximated by $\mathcal{B}$ then applying the operation maintains this approximation by $\mathcal{B}$.

Definition 3.6 Suppose op is an arity $k$ operation with universe $(\mathcal{A}, A), \mathcal{B}$ is a class of functions with universe $B$, and $\omega: A \rightarrow B$ is an interpretation. We write op $\preceq_{+/-}^{\mathcal{E}, \omega} \mathcal{B}$ to mean: 
For any $f_{1}, \ldots, f_{k} \in \mathcal{A}$, if $f_{1}, \ldots, f_{k} \preceq_{+/-}^{\mathcal{E}, \omega} \mathcal{B}$ then op $\left(f_{1}, \ldots, f_{k}\right) \preceq_{+/-}^{\mathcal{E}, \omega} \mathcal{B}$

The notational conventions for approximation (remark 2.4) continue to apply for the approximation of operations; recall that by convention we can choose "+" throughout or "-" throughout in the above definition. It will be useful here and later to define the notion of restricting an operation to certain functions.

Definition 3.7 Suppose op is an operation on universe $\mathcal{F}$ and $\mathcal{G} \subseteq \mathcal{F}$. Furthermore, suppose that op applied to functions in $\mathcal{G}$ yields a function in $\mathcal{G}$. Then by op $\boldsymbol{p}_{\mathcal{G}}$ we mean the same operation as op, except that its universe is $\mathcal{G}$. We use a subset $X$ of $\mathbb{R}$ in place of $\mathcal{G}$ if we mean for $\mathcal{G}$ to be all the functions with universe $X$.

To make the definition more concrete consider "interpreting" composition.

Proposition 3.8 Suppose $A, B \subseteq \mathbb{R}$ and $\omega: A \rightarrow B$ is an interpretation. Suppose $\mathcal{B}$ is any class of functions with universe $B$, closed under composition. Then comp $p_{\mid A} \preceq[\omega] \mathcal{B}$.

\section{Proof}

Suppose $f(t, \bar{x})$ and $g(\bar{y})$ are functions on universe $A$ and $f^{\omega}(t, \bar{x})$ and $g^{\omega}(\bar{y})$ are functions in $\mathcal{B}$ such that $f \preceq^{\omega} f^{\omega}$ and $g \preceq^{\omega} g^{\omega}$. Since $\mathcal{B}$ is closed under composition, $f^{\omega}\left(g^{\omega}(\bar{y}), \bar{x}\right) \in \mathcal{B}$ and it suffices to show that $f(g(\bar{y}), \bar{x}) \preceq^{\omega} f^{\omega}\left(g^{\omega}(\bar{y}), \bar{x}\right)$. Consider any $\bar{x}, \bar{y} \in A$ and the following calculation finishes the proof:

$$
\begin{aligned}
\omega^{-1} \circ f^{\omega}\left(g^{\omega}(\omega(\bar{y})), \omega(\bar{x})\right) & =\omega^{-1} \circ f^{\omega}(\omega \circ g(\bar{y}), \omega(\bar{x})) \\
& =\omega^{-1} \circ \omega \circ f(g(\bar{y}), \bar{x}) \\
& =f(g(\bar{y}), \bar{x})
\end{aligned}
$$

The first equality follows by $g \preceq^{\omega} g^{\omega}$ and the second by $f \preceq^{\omega} f^{\omega}$.

The following is an easy but repeatedly used lemma.

Lemma 3.9 Suppose $\mathcal{B}_{1}$ and $\mathcal{B}_{2}$ are classes of functions with universes $U_{1}$ and $U_{2}$, respectively, and $\omega: U_{1} \rightarrow U_{2}$ is an interpretation. Suppose $\mathcal{O}_{1}$ and $\mathcal{O}_{2}$ are sets of operations whose universes are $\left(\mathcal{F}_{1}, U_{1}\right)$ and $\left(\mathcal{F}_{2}, U_{2}\right)$, respectively, for some $\mathcal{F}_{1}$ and $\mathcal{F}_{2}$.

$$
\begin{aligned}
& \text { If } \mathcal{B}_{1} \preceq_{+/-}^{\mathcal{E},[\omega]} F A\left[\mathcal{B}_{2} ; \mathcal{O}_{2}\right] \text { and for every op } \in \mathcal{O}_{1} \text { we have op } \preceq_{+/-}^{\mathcal{E},[\omega]} \text { FA[ }\left[\mathcal{B}_{2} ; \mathcal{O}_{2}\right] \text { then } \\
& F A\left[\mathcal{B}_{1} ; \mathcal{O}_{1}\right] \preceq_{+/-}^{\mathcal{E},[\omega]} F A\left[\mathcal{B}_{2} ; \mathcal{O}_{2}\right] .
\end{aligned}
$$

\section{Proof}

We show inductively on $f \in \mathrm{FA}\left[\mathcal{B}_{1} ; \mathcal{O}_{1}\right]$ that $f \preceq_{+/-}^{\mathcal{E},[\omega]} \operatorname{FA}\left[\mathcal{B}_{2} ; \mathcal{O}_{2}\right]$. For the basic functions $\mathcal{B}_{1}$ we are given that fact. For any other function op $\left(f_{1}, \ldots, f_{k}\right) \in \mathrm{FA}\left[\mathcal{B}_{1} ; \mathcal{O}_{1}\right]$, we induc-

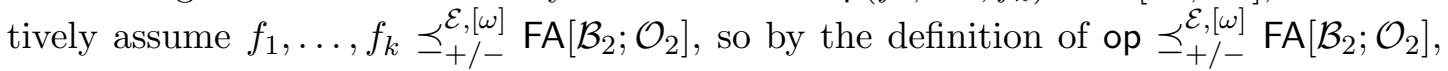

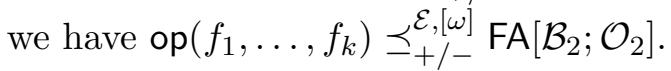


The previous lemma demonstrates the utility of approximating an operation. The straightforward approach to showing that some function algebra contains another (or approximates another) is to work inductively on the particular function algebra in question. For another related claim, the same process is carried out, typically starting from scratch. With the concept of approximating an operation we can show once and for all the resources needed to approximate an operation and then this fact can be re-used in different contexts (e.g. proposition 3.8 is a simple case showing that the interpreting class only needs to be closed under composition). This technical point fits in with our vision of developing a collection of generally applicable tools, within the context of our method of approximation. However, in many cases, to keep the work simpler, we have not worried about limiting the resources to the least necessary.

We will want to show how composition can be approximated in a general way (in this paper it will be used for two special cases). The approximation will use the concept of modulus functions, which will appear throughout the paper. These functions enforce a strong notion of continuity. It is well-known that the functions of Computable Analysis are continuous on their domain, but they have a stronger property of having modulus functions, which witness the continuity. We modify the usual notion to allow the input $\bar{x}$ to function $f(\bar{x})$ to also be input to the modulus function, in addition to the usual "accuracy parameter" (Ko [7], for example, usually works over a compact domain and so the modulus function for $f(\bar{x})$ is a function $m(z)$ with just one argument, an accuracy parameter).

\section{Definition 3.10}

- $|\bar{b}-\bar{a}|$ abbreviates $\left|b_{1}-a_{1}\right|+\ldots+\left|b_{n}-a_{n}\right|$.

- Suppose $f(\bar{x})$ and $m(\bar{x}, z)$ are functions in which the universe of $f$ is contained in the universe of $m$. Then $m$ is a modulus for $f$ if the following two conditions hold:

- $m$ is decreasing and $m(\bar{x}, z)>0$.

- For all $\bar{x}$ and $\bar{y}$ in the universe of $f$, and $z>0$ in the universe of $m,|\bar{x}-\bar{y}| \leq m(\bar{x}, z)$ implies $|f(\bar{x})-f(\bar{y})| \leq 1 / z$.

- A class of functions $\mathcal{M}$ is a modulus for the class of functions $\mathcal{F}$ if for any $f \in \mathcal{F}$, there is $m \in \mathcal{M}$ such that $m$ is a modulus for $f$.

The particular definition of a modulus function is motivated by the following definition.

Definition 3.11 We say a function on universe $\mathbb{Q}$ is continuous if it can be extended to a continuous function on universe $\mathbb{R}$.

From the definitions we can conclude the following.

Proposition 3.12 If $f$ is a $\mathbb{Q}$-function which has a modulus, then $f$ is continuous.

Now we show how the composition operation can be approximated.

Lemma 3.13 Let $\mathcal{H}$ and $\mathcal{F}$ be a classes of functions closed under composition and let $\mathcal{B}$ be a bounding class.

If $\mathcal{H} \leq \mathcal{B}$ and $\mathcal{H}$ has a $1 / \mathcal{B}$ modulus then comp $_{\mid \mathcal{H}} \preceq_{+}^{1 / \mathcal{B}} \mathcal{F}$. 


\section{Proof}

Suppose $f(u), g(x) \in \mathcal{H}$ (one variable for simplicity) and $h(x)=f(g(x))$. For $r(x, \bar{y}) \in$ $\mathcal{B}$, we need $h^{*}(x, \bar{y}) \in \mathcal{F}$ such that $h \preceq^{1 / r} h^{*}$. We will choose $\alpha_{1}, \alpha_{2} \in \mathcal{B}$ and let $f^{*}, g^{*} \in \mathcal{F}$ such that $f \preceq^{1 / \alpha_{1}} f^{*}$ and $g \preceq^{1 / \alpha_{2}} g^{*}$, and define $h^{*}$ by composing them.

Suppose the modulus of $f(u)$ is $1 / m(u, z)$ for $m \in \mathcal{B}$. Suppose $|g(x)| \leq b(x)$ for $b \in \mathcal{B}$. Let $\alpha_{1}(u, x, \bar{y}) \in \mathcal{B}$ such that $2 r(x, \bar{y}) \leq \alpha_{1}(u, x, \bar{y})$. Let $\alpha_{2}(x, \bar{y}) \in \mathcal{B}$ such that $m(b(x), 2 r(x, \bar{y})) \leq \alpha_{2}(x, \bar{y})$. Note that $\alpha_{1}, \alpha_{2} \in \mathcal{B}$ by the properties of bounding classes.

Suppose $\left|g(x)-g^{*}(x, \bar{y})\right| \leq 1 / \alpha_{2}(x, \bar{y})$ and $\left|f(u)-f^{*}(u, x, \bar{y})\right| \leq 1 / \alpha_{1}(u, x, \bar{y})$. Let $h^{*}(x, \bar{y})=f^{*}\left(g^{*}(x, \bar{y}), x, \bar{y}\right)$. Now we show $h \preceq^{1 / r} h^{*}$.

$$
\begin{aligned}
\left|h(x)-h^{*}(x, \bar{y})\right| & \leq\left|f(g(x))-f\left(g^{*}(x, \bar{y})\right)\right|+\left|f\left(g^{*}(x, \bar{y})\right)-f^{*}\left(g^{*}(x, \bar{y}), x, \bar{y}\right)\right| \\
& \leq \frac{1}{2 r(x, \bar{y})}+\frac{1}{\alpha_{1}\left(g^{*}(x, \bar{y}), x, \bar{y}\right)} \\
& \leq \frac{1}{r(x, \bar{y})}
\end{aligned}
$$

Note that we obtain the second inequality by using the modulus on $f$ and the definitions of $\alpha_{1}$ and $\alpha_{2}$.

\section{The Elementary Computable Functions: Applying Approximation}

In this section we apply the ideas of approximation to reprove a result from [3], which says that the "discrete part" of $\mathcal{L}$, a set of $\mathbb{R}$-functions, is exactly the elementary computable functions on $\mathbb{N}$.

Definition 4.1 (from [3]) Suppose $\mathcal{F}$ is a class of functions on $\mathbb{R}$. We let

$$
d p(\mathcal{F})=\left\{f_{\mid \mathbb{N}} \mid f \in \mathcal{F} \text { and } f_{\mid \mathbb{N}} \text { has codomain } \mathbb{N}\right\},
$$

calling this the discrete part of $\mathcal{F}$.

The key analog operation on $\mathbb{R}$ is the operation of obtaining a solution to a linear differential equation.

Definition $4.2 \mathrm{LI}$ is the operation which takes any $g_{1}(\bar{x}), \ldots, g_{n}(\bar{x}), s_{11}(y, \bar{x}), \ldots, s_{n n}(y, \bar{x})$, and returns $h_{1}(y, \bar{x})$ where we have the following defining equations:

$$
\begin{aligned}
& h_{1}(0, \bar{x})=g_{1}(\bar{x}) \\
& \vdots \\
& h_{n}(0, \bar{x})=g_{n}(\bar{x})
\end{aligned}
$$




$$
\begin{aligned}
& \frac{\partial}{\partial y} h_{1}(y, \bar{x})=s_{11}(y, \bar{x}) h_{1}(y, \bar{x})+\ldots+s_{1 n}(y, \bar{x}) h_{n}(y, \bar{x}) \\
& \vdots \\
& \frac{\partial}{\partial y} h_{n}(y, \bar{x})=s_{n 1}(y, \bar{x}) h_{1}(y, \bar{x})+\ldots+s_{n n}(y, \bar{x}) h_{n}(y, \bar{x})
\end{aligned}
$$

Note that technically $\mathrm{LI}$ is not an operation in our sense because it does not have a fixed arity; we can simply view it as a convenient way to refer to a set of operations, each having a fixed arity. For $k \in \mathbb{N}$, by $\mathcal{C}^{k}$ we mean the $k$-times continuously differentiable functions on $\mathbb{R}$. The following basic functions are all $\mathcal{C}^{2}$.

Definition 4.3 Let basic $_{\mathbb{R}}$ be the following functions with universe $\mathbb{R}: 0,1,-1, \pi, P, \theta_{3}$, where $P$ is the set of all projection functions on $\mathbb{R}$ (note that independent of the universe, we use the same notation for projection functions), $\pi$ is the famous constant, and for any $k \in \mathbb{N}(k>0)$, $\theta_{k}(x)=\left\{\begin{array}{ll}0, & x<0 ; \\ x^{k}, & x \geq 0 .\end{array}, a \mathcal{C}^{k-1}\right.$ version of the discontinuous function which indicates whether $a$ number is to the left or right of zero.

The function algebra on the reals that we will now be concerned with is the following (using the notation $\mathcal{L}$ from earlier papers).

Definition 4.4 Let $\mathcal{L}$ abbreviate the function algebra $F A\left[\right.$ basic $_{\mathbb{R}} ;$ comp, $L I$.

The goal we are now aiming for is theorem 4.26:

$$
\operatorname{dp}(\mathcal{L})=\mathrm{FA}_{\mathbb{N}} .
$$

The proof in [3] proceeds by showing the two inclusions. The inclusion " $\supseteq$ " (proposition 4.8 of [3]) is proved inductively on the construction of the functions in $\mathrm{FA}_{\mathbb{N}}$, using the operations of $\mathcal{L}$ at each step. The inclusion " $\subseteq$ " (proposition 4.4 of [3]) is again proved by induction, this time on the functions in $\mathcal{L}$, but rather than using the operations of $F A_{\mathbb{N}}$ at each step, a Turing Machine is constructed, and it is shown how in elementary time an appropriately close approximation can be carried out; of course this relies on the well-known fact that the function algebra $F A_{\mathbb{N}}$ corresponds to the elementary time computable functions. We will give an alternative proof of this inclusion using our method of approximation. Other than using the fact that $F A_{\mathbb{N}}$ contains a few useful functions (being a well known and powerful class), the proof will proceed naturally using the operations of the function algebra itself, without reference to the elementary computable functions. Furthermore, we re-use the main approximation results of this section (namely corollaries 4.14 and 4.25 ) in proving the main theorem of the next section.

If one were to begin thinking about a proof along these lines, an apparent problem presents itself. A function $f \in \operatorname{dp}(\mathcal{L})$ is in there due to some associated construction tree (recall definition 3.5). While $f$ (the function associated with the root of the construction tree) is required to have natural number values on natural number inputs, there is no such constraint on the functions associated with other nodes in the construction tree (they may be real valued). To inductively show that $f$ is in $\mathrm{FA}_{\mathbb{N}}$, requires that we deal with these non-root nodes in $\mathrm{FA}_{\mathbb{N}}$; however, it is unclear how to deal with real number values in $\mathrm{FA}_{\mathbb{N}}$. The way we get around this issue is to introduce an intermediary function algebra with universe $\mathbb{Q}$. This function algebra will naturally approximate $\mathcal{L}$ (corollary 4.25). Then we can naturally interpret this function algebra on $\mathbb{Q}$ into $\mathrm{FA}_{\mathbb{N}}$ (corollary 4.14). The theorem then follows, using the transitivity of the approximation relation. 
The main operations of the function algebra on $\mathbb{Q}$ will be a kind of bounded sum (line $\sum$ ) and bounded product (line $\prod$ ) on the rationals. They are defined so that they preserve continuous functions when applied to continuous functions. This property is important for the next section, and while not important for this section, presents little complication for it. We call the operation a line sum because it is defined as the linearization of summation. For the definition, given a $\mathbb{Q}$-function $f(y, \bar{x})$, we understand $g(z, \bar{x})=\sum_{y=0}^{z} f(y, \bar{x})$ to be defined in the natural way for negative $z \in \mathbb{Z}$, e.g. $\sum_{y=0}^{-2} f(y, \bar{x})=f(0, \bar{x})+f(-1, \bar{x})+f(-2, \bar{x})$. Furthermore, we understand $g(z, \bar{x})$ to be a function with values in $\mathbb{Q}$ where $z \in \mathbb{Z}$ and $\bar{x} \in \mathbb{Q}$. The discussion for products is similar.

Definition 4.5 We define operations line $\sum$ and line $\prod$ with universe $\mathbb{Q}$. Suppose $f(y, \bar{x})$ is a function on universe $\mathbb{Q}$.

- line $\sum(f)=\operatorname{Lin}\left(\sum_{y=0}^{z} f(y, \bar{x}) ; z\right)$

$$
\left(\text { which }=(1+\lfloor z\rfloor-z) \sum_{y=0}^{\lfloor z\rfloor} f(y, \bar{x})+(z-\lfloor z\rfloor) \sum_{y=0}^{\lceil z\rceil} f(y, \bar{x})\right)
$$

- line $\prod(f)=\operatorname{Lin}\left(\prod_{y=0}^{z} f(y, \bar{x}) ; z\right)$.

We sometimes write line $\sum(f)$ as line $\sum_{y=0}^{z} f(y, \bar{x})$ to express how we use variables, and likewise for line $\prod$.

Definition 4.6 Let basic $\mathbb{Q}$ be the following functions with universe $\mathbb{Q}: 0,1,-1, P, *$, , div, $\theta_{1}$, where $P$ is the set of projection functions, $\theta_{1}$ is understood as a function with universe $\mathbb{Q}$ (though it was originally defined for $\mathbb{R}$ in definition 4.3), and $\operatorname{div}(x)= \begin{cases}1 / x, & \text { if } x \geq 1 ; \\ 1, & \text { otherwise. }\end{cases}$

Definition 4.7 Let the function algebra $F A\left[\right.$ basic $_{\mathbb{Q}}$; comp, line $\sum$, line $\left.\prod\right]$ be abbreviated by $F A_{\mathbb{Q}}(c t n)$.

Notice that all the functions in this class are continuous as indicated by "ctn". We explicitly indicate this because in the next section we will define an extension of this function algebra which contains discontinuous functions (it will be called $\mathrm{FA}_{\mathbb{Q}}$ (disctn)).

If it appears to you that the basic functions are redundant, you are probably correct. We should be able to derive $*$ and + in the class, as is typically done for these functions in $\mathrm{FA}_{\mathbb{N}}$. However, for us the classes on the rationals are merely a means to an end, so we include possible redundancy to simplify the technical development. We define some functions contained in $\mathrm{FA}_{\mathbb{Q}}$ (ctn).

Definition 4.8 We define some functions with universe $\mathbb{Q}$.

- Let $\operatorname{sgn}(x)= \begin{cases}0, & \text { if } x \leq 0 ; \\ x, & \text { if } 0<x<1 ; \\ 1, & \text { if } x \geq 1 .\end{cases}$

- Let $\delta(x)= \begin{cases}1-x, & 0 \leq x \leq 1 \\ 1+x, & -1 \leq x \leq 0 \\ 0, & |x| \geq 1\end{cases}$

- Let $\delta_{u}(x)=\delta(x-u)$ 
Since $\operatorname{sgn}(x)=\theta_{1}(x)-\theta_{1}(x-1)$, it is in $\mathrm{FA}_{\mathbb{Q}}(\mathrm{ctn})$. We could continue to show various simple functions (like $\delta$ ) are in $\mathrm{FA}_{\mathbb{Q}}(\operatorname{ctn})$, but if we only care about the values on $\mathbb{N}$, the following simple proposition will be useful. It says in words, that $F A_{\mathbb{Q}}(\mathrm{ctn})$ can exactly capture $F A_{\mathbb{N}}$, when restricted to $\mathbb{N}$.

Proposition $4.9 \quad F A_{\mathbb{N}} \preceq F A_{\mathbb{Q}}(c t n)$

\section{Proof}

Note that we can define cut-off subtraction in $\mathrm{FA}_{\mathbb{Q}}(\operatorname{ctn})$ by $x-y=\operatorname{sgn}(x-y)(x-y)$; the other basic functions can likewise be evaluated, thus we have basic $\mathbf{b}_{\mathbb{N}} \preceq \mathrm{FA}_{\mathbb{Q}}$ (ctn). Immediately from the definitions of line sums and line products, we have $\sum, \prod \preceq$ $\mathrm{FA}_{\mathbb{Q}}(\mathrm{ctn})$, thus by lemma 3.9 we are done.

Since we can essentially work with $\mathbb{Z}$ using $\mathbb{N}$ we will sometimes reference the previous proposition for functions with universe $\mathbb{Z}$. We can now conclude that $F A_{\mathbb{Q}}$ (ctn) contains a function that looks like $\delta$ on $\mathbb{Z}$; to get exactly $\delta$, we can linearize (recall definition 2.8), which is allowed in $\mathrm{FA}_{\mathbb{Q}}$ (ctn) by the next lemma.

Lemma 4.10 $F A_{\mathbb{Q}}($ ctn) is closed under Lin.

\section{Proof}

Suppose $f\left(x_{1}, \ldots, x_{k}\right)$ is in $\mathrm{FA}_{\mathbb{Q}}(\mathrm{ctn})$. Note that Lin has the following property (follows by writing out the definition):

$$
\text { Suppose } r<k \text {. Then } \operatorname{Lin}\left(\operatorname{Lin}\left(f ; x_{1}, \ldots, x_{r}\right) ; x_{r+1}\right)=\operatorname{Lin}\left(f ; x_{1}, \ldots, x_{r+1}\right) .
$$

Due to this property it suffices to just show that $\operatorname{Lin}\left(f ; x_{1}\right)$ is in $\mathrm{FA}_{\mathbb{Q}}(\mathrm{ctn})$. Thus we just note that $\operatorname{Lin}\left(f ; x_{1}\right)=$ line $\sum_{y=0}^{x_{1}} f\left(y, x_{2}, \ldots, x_{k}\right) \delta_{x_{1}}(y)$.

Now we point out a series of facts, leading to corollary 4.14 , which states that $\mathrm{FA}_{\mathbb{Q}}$ (ctn) can be interpreted in $\mathrm{FA}_{\mathbb{N}}$. Example 2.7 in fact shows how multiplication in basic $\mathbb{Q}_{\mathbb{Q}}$ can be interpreted (via $\lambda$ ) in $\mathrm{FA}_{\mathbb{N}}$; the other functions of basic $_{\mathbb{Q}}$ can be handled similarly, thus we have the following lemma.

Lemma 4.11 basic $_{\mathbb{Q}} \preceq^{\lambda} F A_{\mathbb{N}}$

Lemma 4.12 line $\prod \preceq^{\lambda} F A_{\mathbb{N}}$

\section{Proof}

Suppose $f(y)$ is a function (we ignore arguments other than $y$ ) with universe $\mathbb{Q}$ and $h(z)=\prod_{y=0}^{\lfloor z\rfloor} f(y)$. Assuming we have $f^{\lambda}(y) \in \mathrm{FA}_{\mathbb{N}}$ such that $f \preceq^{\lambda} f^{\lambda}$, we will find $h^{\lambda}(z) \in \mathrm{FA}_{\mathbb{N}}$ such that $h \preceq^{\lambda} h^{\lambda}$, i.e. $h(z)=\lambda^{-1} \circ h^{\lambda}(\lambda(z))$. To interpret line $\prod$ then involves interpreting $\prod_{y=0}^{[z\rceil} f(y)$ (which is similar to finding $h^{\lambda}$ ) and then putting this 
interpretation together with $h^{\lambda}$. Defining $h^{\lambda}$ will be similar to interpreting multiplication in example 2.7 (we will again use the functions from definition 2.6). To find the bounded product, we will find what the top and bottom of the resulting fraction should be, along with its sign and put this together properly.

Consider the following $\mathbb{N}$-functions:

$$
\begin{aligned}
& \operatorname{TOP}(w)=\prod_{y=0}^{w} \text { top } \circ f^{\lambda} \circ \lambda_{\mid \mathbb{N}}(y) \\
& \operatorname{BOT}(w)=\prod_{y=0}^{w} \text { bot } \circ f^{\lambda} \circ \lambda_{\mid \mathbb{N}}(y) \\
& \operatorname{SIGN}(w)=\text { parity }\left\{\sum_{y=0}^{w} \text { parity } \circ f^{\lambda} \circ \lambda_{\mid \mathbb{N}}(y)\right\}
\end{aligned}
$$

Note that

$$
h(z)=\operatorname{SIGN}(\lfloor z\rfloor) \frac{\operatorname{TOP}(\lfloor z\rfloor)}{\operatorname{BOT}(\lfloor z\rfloor)}
$$

for $z>0$ (we are ignoring the case of $z<0$, which could be handled by using a function which chooses the case depending on the sign of $z$ ). Since $\lambda_{\mid \mathbb{N}}, f^{\lambda} \in \mathrm{FA}_{\mathbb{N}}$, so are TOP, BOT, and SIGN. Let $b \in \mathrm{FA}_{\mathbb{N}}$ such that $b(\lambda(z))=\lfloor z\rfloor$, so

$$
h(z)=\operatorname{SIGN}(b(\lambda(z))) \frac{\operatorname{TOP}(b(\lambda(z)))}{\operatorname{BOT}(b(\lambda(z)))} .
$$

We let $h^{\lambda}(u)=\operatorname{code}(\operatorname{TOP}(b(u)), \operatorname{BOT}(b(u)), \operatorname{SIGN}(b(u))) \in \mathrm{FA}_{\mathbb{N}}$, and thus $h^{\lambda}(\lambda(z))$ is the code of $h(z)$ and $\lambda^{-1} \circ h^{\lambda}(\lambda(z))$ is as desired.

The proof for sums is similar, though finding the "top" is a bit more technically involved.

Lemma 4.13 line $\sum \preceq^{\lambda} F A_{\mathbb{N}}$

Corollary 4.14FA $F A_{\mathbb{Q}}($ ctn $) \preceq^{\lambda} F A_{\mathbb{N}}$

\section{Proof}

By lemma 3.9, it suffices to show that basic $_{Q} \preceq^{\lambda} \mathrm{FA}_{\mathbb{N}}$, and that $\mathrm{FA}_{\mathbb{N}}$ interprets the 3 operations in $\mathrm{FA}_{\mathbb{Q}}$ (ctn). The last two lemmas showed that both line sums and products can be interpreted. Proposition 3.8 shows that composition can be interpreted.

The rest of the section is mostly devoted to the goal of proving corollary 4.25 , which states that we can approximate $\mathcal{L}$ by $\mathrm{FA}_{\mathbb{Q}}(\mathrm{ctn})$. Approximating the basic functions of $\mathcal{L}$ is relatively straightforward, as is the following bound.

Proposition 4.15 $\mathcal{T W} \leq F A_{\mathbb{Q}}(c t n)$.

Lemma 4.16 basic $_{\mathbb{R}} \preceq_{+}^{1 / \mathcal{T W}} F A_{\mathbb{Q}}($ ctn $)$

Proof 
Except for $\theta_{3}$ and the constant $\pi$, all the functions and constants of $\mathbf{b a s i c}_{\mathbb{R}}$ are extensions of something in basic e $_{\mathbb{Q}}$ and so we approximate them with zero error on $\mathbb{Q}$. We can approximate $\theta_{3}$ with zero error since $\theta_{3}=\theta_{1} * \theta_{1} * \theta_{1}$. For $\pi$ we carry out a sufficiently long Taylor series approximation, which is simulated using line $\sum$ and other simple functions from $\mathrm{FA}_{\mathbb{Q}}$ (ctn); notice the importance of div to express the Taylor sum. The necessary length of the series will be a function from $\mathcal{T} \mathcal{W}$, which we can dominate in $\mathrm{FA}_{\mathbb{Q}}(\mathrm{ctn})$, by proposition 4.15 .

We will want to use Euler's Method in $\mathrm{FA}_{\mathbb{Q}}$ (ctn) to approximate solutions to differential equations; to carry this out, linear recursion will be a useful tool (we begin with the definition on $\mathbb{N})$.

Definition 4.17 $L R$ is the operation which takes any functions on universe $\mathbb{N}, g_{1}(\bar{x}), \ldots, g_{n}(\bar{x})$, $s_{11}(y, \bar{x}), \ldots, s_{n n}(y, \bar{x}), t_{1}(y, \bar{x}), \ldots, t_{n}(y, \bar{x})$ and returns $h_{1}(y, \bar{x})$, where we have the following equations:

$$
\begin{aligned}
& h_{1}(0, \bar{x})=g_{1}(\bar{x}) \\
& \quad \vdots \\
& h_{n}(0, \bar{x})=g_{n}(\bar{x}) \\
& \quad h_{1}(y+1, \bar{x})=s_{1 n}(y, \bar{x}) h_{1}(y, \bar{x})+\ldots+s_{1 n}(y, \bar{x}) h_{n}(y, \bar{x})+t_{1}(y, \bar{x}) \\
& \quad \vdots \\
& h_{n}(y+1, \bar{x})=s_{n 1}(y, \bar{x}) h_{1}(y, \bar{x})+\ldots+s_{n n}(y, \bar{x}) h_{n}(y, \bar{x})+t_{n}(y, \bar{x})
\end{aligned}
$$

Special cases of LR yield the operations $\sum$ and $\prod$. It is relatively straightforward to see that we can also obtain LR with $\sum$ and $\prod$.

Proposition 4.18 $F A_{\mathbb{N}}$ is closed under $L R$.

We define a linearized version of linear recursion for the rationals, as we did with sums and products.

Definition 4.19 lineLR is the operation with universe $\mathbb{Q}$ which takes some input functions $f_{1}, f_{2}, \ldots$ and returns:

$$
h(y, \bar{x})=\operatorname{Lin}\left(L R^{*}\left(f_{1}, f_{2}, \ldots\right) ; y\right),
$$

where $y$ is the recursion variable and we understand $L R^{*}$ to be defined just like $L R$ except that the functions $f_{i}$ can have values in $\mathbb{Q}$, and for $y<0$ (where the result of $L R$ would not be defined) we simply define the result of $L R^{*}$ to be zero.

The following lemma can be seen as a kind of "lifting" of proposition 4.18 to an analogous claim on the rationals. 
Lemma 4.20 $F A_{\mathbb{Q}}(c t n)$ is closed under lineLR.

\section{Proof}

We will use the following technical claim (note that we restrict to $\lambda_{\mid \mathbb{N}}$ since it is not possible for the discontinuous function $\lambda$ to be in $\left.\mathrm{FA}_{\mathbb{Q}}(\mathrm{ctn})\right)$ :

$$
\mathrm{FA}_{\mathbb{Q}}(\operatorname{ctn}) \text { contains extensions }(\text { to } \mathbb{Q}) \text { of } \lambda^{-1} \text { and } \lambda_{\mid \mathbb{N}} \text {. }
$$

For the claim, note the following:

- $\lambda_{\mid \mathbb{N}} \in \mathrm{FA}_{\mathbb{N}}$, so by proposition 4.9 we have an extension in $\mathrm{FA}_{\mathbb{Q}}$ (ctn).

- For $\lambda^{-1}$, it suffices to show we can compute top and bot (recall definition 2.6). Consider the main part of top, showing that the bijection $p: \mathbb{N} \rightarrow \mathbb{N}$, defined by $p((1 / 2)(a+b+1)(a+b)+a)=a$ can be extended to $\mathrm{FA}_{\mathbb{Q}}(\mathrm{ctn})$. The following extension to $\mathbb{Q}$ works in $\mathrm{FA}_{\mathbb{Q}}(\mathrm{ctn})$ :

$$
p(z)=\text { line } \sum_{x=0}^{z} \text { line } \sum_{y=0}^{z} y \delta_{z}((y+x+1)(y+x)+y) .
$$

Now for the lemma, suppose $f_{1}, \ldots, f_{k} \in \mathrm{FA} \mathbb{Q}($ ctn $)$ and let $h=\operatorname{lineLR}\left(f_{1}, \ldots, f_{k}\right)$. By corollary 4.14, we have interpretations $f_{1}^{\lambda}, \ldots, f_{k}^{\lambda} \in \mathrm{FA}_{\mathbb{N}}$. By proposition 4.18 , we can use $\mathrm{LR}$ in $\mathrm{FA}_{\mathbb{N}}$, and show that $h^{\lambda} \in \mathrm{FA}_{\mathbb{N}}$ and so by proposition 4.9 we have an extension of it in $\mathrm{FA}_{\mathbb{Q}}(\mathrm{ctn})$. By the above claim, we have $\lambda_{\mid \mathbb{N}}, \lambda^{-1} \in \mathrm{FA}_{\mathbb{Q}}(\mathrm{ctn})$ and so on the naturals, $h=\lambda^{-1} \circ h^{\lambda} \circ \lambda_{\mid \mathbb{N}} \in \mathrm{FA}_{\mathbb{Q}}$ (ctn); to finish we linearize, allowed by proposition 4.10.

To apply Euler's Method we will need appropriate bounds on the functions used.

Definition 4.21 Suppose $\mathcal{F}$ is a class of differentiable functions. Then $\mathcal{F}^{\prime}=\left\{h^{\prime} \mid h \in \mathcal{F}\right\}$, where by $h^{\prime}$ we mean that $h$ is differentiated with respect to any one variable.

The following is proved inductively (observed in proposition 4.3 of [3]).

Proposition $4.22 \mathcal{L}, \mathcal{L}^{\prime} \leq \mathcal{T} \mathcal{W}$

The last proposition allows us to conclude the following (since if the derivative of a function is bounded by $\mathcal{T} \mathcal{W}$, there is a $1 / \mathcal{T} \mathcal{W}$ modulus).

Corollary $4.23 \mathcal{L}$ has a $1 / \mathcal{T} \mathcal{W}$ modulus.

Since we are concerned with $\mathrm{LI}$ in $\mathcal{L}$, restricting our attention to $\mathrm{LI}_{\mid \mathcal{L}}$ is fine; in fact to prove the next lemma, we could replace $\mathcal{L}$ by any class of $\mathcal{C}^{2}$ functions $\mathcal{F}$ satisfying $\mathcal{F}, \mathcal{F}^{\prime} \leq \mathcal{T} \mathcal{W}$.

Lemma 4.24 $L I_{\mid \mathcal{L}} \preceq_{+}^{1 / \mathcal{T W}} F A_{\mathbb{Q}}(c t n)$

Proof 
Suppose $f_{1}, f_{2}, g_{1}, g_{2} \in \mathcal{L}$ and we use $\mathbf{L I}$ to define $h_{1}$ via the following linear differential equations (for ease of readability, we just consider 2 equations):

$$
\begin{aligned}
& \frac{\partial}{\partial x} h_{1}(x, \bar{y})=f_{1}(x, \bar{y}) h_{1}(x, \bar{y})+f_{2}(x, \bar{y}) h_{2}(x, \bar{y})=F_{1}\left(x, h_{1}, h_{2}\right) \\
& \frac{\partial}{\partial x} h_{2}(x, \bar{y})=g_{1}(x, \bar{y}) h_{1}(x, \bar{y})+g_{2}(x, \bar{y}) h_{2}(x, \bar{y})=F_{2}\left(x, h_{1}, h_{2}\right)
\end{aligned}
$$

Suppose that $1 / \alpha(x, \bar{y}, \bar{z}) \in 1 / \mathcal{T} \mathcal{W}$ is the desired approximation accuracy, and we need to find $h_{1}^{*}(x, \bar{y}, \bar{z}) \in \mathrm{FA}_{\mathbb{Q}}(\mathrm{ctn})$ such that $h_{1} \preceq^{1 / \alpha} h_{1}$.

The function $h_{1}^{*}$ will be defined by essentially simulating Euler's method, using sufficiently good approximations $f_{1}^{*}, f_{2}^{*}, g_{1}^{*}$, and $g_{2}^{*}$ of $f_{1}, f_{2}, g_{1}$, and $g_{2}$, respectively. The simulation will be carried out on the interval $[0, x]$, using the partition $0=x_{0}<x_{1}<$ $\ldots<x_{n}=x$, where $\left|x_{i+1}-x_{i}\right|=\delta(x, \bar{y}, \bar{z})=1 / b(x, \bar{y}, \bar{z})$, for a function $\delta \in \mathrm{FA}_{\mathbb{Q}}$ (ctn) that goes to 0 quickly enough ( $b$ is a fast growing function in $\mathrm{FA}_{\mathbb{Q}}$ (ctn), larger than 1 , so that $\left.\delta=\operatorname{div}(b) \in \mathrm{FA}_{\mathbb{Q}}(\operatorname{ctn})\right)$. Note that we only consider $x>0$, but for $x<0$, we would be able to carry out the same procedure and we could then use a function in $\mathrm{FA}_{\mathbb{Q}}$ (ctn) to choose the procedure, depending on the sign of $x$. To carry this out we use lineLR to obtain $h_{1}^{*}$ from the following system of equations (we drop the parameters $\bar{y}, \bar{z}$ ).

$$
\begin{aligned}
& h_{1}^{*}(u+1)=h_{1}^{*}(u)+\delta(x)\left\{f_{1}^{*}(u \delta(x)) h_{1}^{*}(u)+f_{2}^{*}(u \delta(x)) h_{2}^{*}(u)\right\} \\
& h_{2}^{*}(u+1)=h_{2}^{*}(u)+\delta(x)\left\{g_{1}^{*}(u \delta(x)) h_{1}^{*}(u)+g_{2}^{*}(u \delta(x)) h_{2}^{*}(u)\right\}
\end{aligned}
$$

Notice that we use a new variable $u$ to keep track of the steps in Euler's Method. The function $\delta$ keeps $x$ as its input since to obtain the desired accuracy, the partition depends on the final point, $x$. The other functions have appropriate parameters substituted, so that the value of $h_{1}^{*}(u)$ is the approximation of $h_{1}\left(x_{u}\right)$. Thus $h_{1}^{*}(x \cdot b(x, \bar{y}, \bar{z}))$ approximates $h_{1}(x)$. Note that the arithmetic functions,$+ *$, and div are sufficient to define these equations (in particular, div is essential).

To check that the error really is bounded by $1 / \mathcal{T} \mathcal{W}$, we follow the standard error analysis for Euler's method. Consider some fixed $x, \bar{y}, \bar{z}$ and the partition of $[0, x]$ discussed above. In the usual way we write

$$
\begin{aligned}
& h_{1}\left(x_{i+1}\right)=h_{1}\left(x_{i}\right)+\delta F_{1}\left(x_{i}, h_{1}, h_{2}\right)+\delta \tau \\
& h_{2}\left(x_{i+1}\right)=h_{2}\left(x_{i}\right)+\delta F_{2}\left(x_{i}, h_{1}, h_{2}\right)+\delta \tau,
\end{aligned}
$$

where the local error $\tau$ can be bound by $\delta d$, where $d$ is a bound on $h_{1}^{\prime \prime}(x)$ and $h_{2}^{\prime \prime}(x)$. Since the $f_{i}$ and $g_{i}$ from which $h_{1}$ and $h_{2}$ are defined have $\mathcal{T W}$ bounds, so do $h_{1}^{\prime \prime}(x)$ and $h_{2}^{\prime \prime}(x)$. Because $F_{1}$ and $F_{2}$ are defined in terms of $f_{i}$ and $g_{i}$ with $\mathcal{T} \mathcal{W}$ bounds, there is $L(x, \bar{y}, \bar{z}) \in \mathcal{T} \mathcal{W}$ which is a Lipshitz function for $F_{1}$ and $F_{2}$, i.e. for $u \in[0, x]$, we have $\left|F_{1}\left(u, h_{1}^{(2)}, h_{2}^{(2)}\right)-F_{1}\left(u, h_{1}^{(1)}, h_{2}^{(1)}\right)\right| \leq L\left(\left|h_{1}^{(2)}-h_{1}^{(1)}\right|+\left|h_{2}^{(2)}-h_{2}^{(1)}\right|\right)$, and likewise for $F_{2}$. The global error can then be calculated recursively in the usual way (i.e. the error $e_{i+1}$ at step $i+1$ is calculated in terms of the error at step $\left.i, e_{i}=\left|h_{1}\left(x_{i}\right)-h_{1}^{*}(i)\right|\right)$. We arrive at the error bound of $e^{x L}\left(\left|e_{0}\right|+\delta d+r\right)$, where $r$ is the error incurred by using the approximation to the derivative at each step in Euler's method, as opposed to the exact derivative (i.e. we use $f_{i}^{*}$ and $g_{i}^{*}$ rather than $f_{i}$ and $g_{i}$ to define the $h_{i}^{*}$ ). We now 
see that we can make this error less than $1 / \alpha$ because starting with $\mathcal{T} \mathcal{W}$ bounds on $L$ and $d$, we can choose arbitrarily good $1 / \mathcal{T} \mathcal{W}$ bounds on $r, \delta$, and $\left|e_{0}\right|$.

Corollary $4.25 \mathcal{L} \preceq_{+}^{1 / \mathcal{T W}} F A_{\mathbb{Q}}($ ctn $)$

\section{Proof}

By lemma 3.9, it suffices to note that basic $\mathbb{R}_{\mathbb{R}} \preceq_{+}^{1 / \mathcal{T} \mathcal{W}} \mathrm{FA}_{\mathbb{Q}}$ (ctn) (by lemma 4.16), and that $\mathrm{FA}_{\mathbb{Q}}(\mathrm{ctn})$ approximates the 2 operations $\mathrm{LI}_{\mid \mathcal{L}}$ and $\operatorname{comp}_{\mid \mathcal{L}}$. Lemma 4.24 shows we can approximate $\mathrm{LI}_{\mid \mathcal{L}}$. For composition, we can apply lemma 3.13, since we know $\mathcal{L} \leq \mathcal{T} \mathcal{W}$ (proposition 4.22) and $\mathcal{L}$ has a $1 / \mathcal{T} \mathcal{W}$ modulus (corollary 4.23).

We can now finish the alternative proof of the theorem from [3].

Theorem $4.26 d p(\mathcal{L})=F A_{\mathbb{N}}$

\section{Proof}

- $\supseteq$ : This direction is carried out inductively in proposition 4.8 of [3] (we make no modification to the existing proof).

- $\subseteq$ : By corollaries 4.25 and 4.14, respectively, we have:

$$
\mathcal{L} \preceq_{+}^{1 / \mathcal{T} \mathcal{W}} \mathrm{FA}_{\mathbb{Q}}(\mathrm{ctn}) \preceq^{\lambda} \mathrm{FA}_{\mathbb{N}} .
$$

By transitivity we have $\mathcal{L} \preceq_{+}^{1 / \mathcal{T} \mathcal{W}, \lambda} \mathrm{FA}_{\mathbb{N}}$. Let $f(\bar{x}) \in \mathrm{dp}(\mathcal{L})$, and take any $\alpha(\bar{x}, \bar{y}) \in$ $1 / \mathcal{T} \mathcal{W}$, so we have $f^{*}(\bar{x}, \bar{y}) \in \mathrm{FA}_{\mathbb{N}}$ such that $f \preceq^{\alpha, \lambda} f^{*}$. By fixing $\bar{y}$ to a large enough number, we have $\alpha(\bar{x}, \bar{y}) \leq 1 / 3$ for all $\bar{x}$ and can obtain $h(\bar{u}) \in \mathrm{FA}_{\mathbb{N}}$ such that for $\bar{x} \in \mathbb{N},\left|f(\bar{x})-\lambda^{-1} \circ h \circ \lambda(\bar{x})\right| \leq 1 / 3$. Since $f(\bar{x}) \in \mathbb{N}$ for $\bar{x} \in \mathbb{N}$, $f(\bar{x})=$ nearest $(h(\lambda(\bar{x}))) \in \mathrm{FA}_{\mathbb{N}}$, where nearest $(a)$ returns the closest natural to the rational coded by $a$. The last function is in $\mathrm{FA}_{\mathbb{N}}$ because both nearest and $\lambda_{\mid \mathbb{N}}$ are in $F A_{\mathbb{N}}$.

Consider again our alternative proof of $\operatorname{dp}(\mathcal{L}) \subseteq \mathrm{FA}_{\mathbb{N}}$. Despite the fact that we were liberal in assuming that $\mathrm{FA}_{\mathbb{N}}$ contained certain functions, our approach allowed us to work more naturally with the function algebra itself, avoiding the characterization of $\mathrm{FA}_{\mathbb{N}}$ as the elementary computable functions. In fact our methods could be used in a natural way for function algebras which do not have any known corresponding computational classes, highlighting a particular advantage of our approach when working with function algebras.

\section{Connection to Computable Analysis: Applying Lifting}

We will begin by introducing the technical framework, and then we discuss explicitly the idea of lifting in the proofs of the theorems. We will use standard notions from Computable Analysis, as described in Ker-I Ko [7] and Weihrauch [12], though following more closely the former. For the 
most part Ker-I Ko restricts his attention to functions defined on a finite interval, while we consider functions defined on all of $\mathbb{R}$. Thus in this work, the main difference is that a number of notions will depend on both the input value to the function, as well as the usual accuracy parameter (as in, for example, the case of the modulus functions). We will be concerned with the elementary computable functions over $\mathbb{N}, \mathbb{Q}$, and $\mathbb{R}$.

Let $\mathbf{E}(\mathbb{N})$ be the usual elementary computable functions on $\mathbb{N}$, defined via Turing Machines. By $\mathbf{E}(\mathbb{R})$ we mean the total $\mathbb{R}$-functions $f(\bar{x})$ which can be computed to accuracy $1 / n(n \geq 1)$ in time $t(\bar{x}, n)$, where $t \in \mathcal{T} \mathcal{W}$. The real input $\bar{x}$ is given by an oracle which gives $\bar{x}$ to any demanded precision as a dyadic rational (the set of dyadic rationals is denoted $\mathbb{D}$ ); the precision $1 / n$ is given by putting $n$ on the input tape (we call this the accuracy input). Note that we use the approximation of the form $1 / n$ rather than $1 / 2^{n}$, since for elementary computable functions such distinctions have no effect. For $\mathbb{Q}$ we will define a class of functions $\mathbf{E}(\mathbb{Q})$ which essentially treats rationals as pairs of integers. The following definitions of a kind of denominator, numerator, and sign function will be convenient.

Definition 5.1 We define functions from $\mathbb{Q}$ to $\mathbb{Z}$.

- For a rational $(-1)^{s} a / b$ presented in lowest terms, let:

$$
\begin{aligned}
& D\left((-1)^{s} a / b\right)=(-1)^{s} b \\
& N\left((-1)^{s} a / b\right)=(-1)^{s} a
\end{aligned}
$$

(note that $D(0)=N(0)=0$ )

- Let $\operatorname{sign}(x)=\left\{\begin{array}{ll}0, & \text { if } x \leq 0 ; \\ 1, & \text { if } x>0 .\end{array}\right.$, a discontinuous version of sgn from definition 4.8 .

A $\mathbb{Q}$-function $f(x)$ is in $\mathbf{E}(\mathbb{Q})$ if there is an elementary time Turing Machine on $\mathbb{N}$ that computes it in the following sense: On input $x \in \mathbb{Q}$ the machine is given the triple $(|\mathrm{N}(x)|,|\mathrm{D}(x)|, \operatorname{sign}(x))$, and it computes the triple $(|\mathrm{N}(f(x))|,|\mathrm{D}(f(x))|, \operatorname{sign}(f(x)))$; note that the time allowed depends on the length of the representation of $x$ as a triple of natural numbers (for a sequence of inputs $\bar{x}$ we use a sequence of triples). Note that $\mathbf{E}(\mathbb{R})$ contains only continuous functions, while $\mathbf{E}(\mathbb{Q})$ contains discontinuous functions (recall definition 3.11 and the surrounding discussion). In general, if a class of functions contains only continuous functions we call it a continuous class and otherwise we call it a discontinuous class.

We introduce the technique of lifting in this section and apply it to extend a result of Bournez and Hainry [1]. The lifting can be viewed as a two step process which is pictured in the figure on page 24. We begin with the classic result of Grzegorczyk stating that $\mathbf{E}(\mathbb{N})=F A_{\mathbb{N}}$. The first step is to lift this to an equality on the rationals, for which we will use an extension of $F A_{\mathbb{Q}}$ (ctn) to a discontinuous class $F A_{\mathbb{Q}}$ (disctn). It is defined by simply adding $D$ to the basic functions of $F A_{\mathbb{Q}}$ (ctn) (we indicate this addition to the basic functions by placing $D$ after the existing basic functions with a comma separating them).

Definition 5.2 We abbreviate $F A\left[\right.$ basic $_{\mathbb{Q}}, D ;$ comp, line $\sum$, line $\left.\prod\right]$ by $F A_{\mathbb{Q}}$ (disctn).

Using $\mathrm{D}$, and the easily derived $\mathrm{N}$, the class $\mathrm{FA}_{\mathbb{Q}}($ disctn) can break apart a rational into a triple of naturals and then work with these naturals in an elementary computable manner, thus it is much more powerful than $\mathrm{FA}_{\mathbb{Q}}(\mathrm{ctn})$. Recalling proposition 4.9 , we can immediately obtain 
$F A_{\mathbb{N}} \preceq F A_{\mathbb{Q}}$ (disctn), and will in fact reference that proposition with respect to either $F A_{\mathbb{Q}}$ (ctn) or $F A_{\mathbb{Q}}$ (disctn). Also, by noting that $\mathrm{D} \preceq^{\lambda} \mathrm{FA}_{\mathbb{N}}$, we can extend corollary 4.14 to $F A_{\mathbb{Q}}$ (disctn), i.e. $F A_{\mathbb{Q}}$ (disctn) $\preceq^{\lambda} F A_{\mathbb{N}}$. Immediate from the definitions we can conclude the following.

Proposition 5.3 $\mathbf{E}(\mathbb{N}) \preceq \mathbf{E}(\mathbb{Q})$ and $\mathbf{E}(\mathbb{Q}) \preceq^{\lambda} \mathbf{E}(\mathbb{N})$.

The relationships between the function classes on the naturals and the (discontinuous) ones on the rationals are pictured in the top of the figure. Note that $\mathbf{E}(\mathbb{Q}) \approx^{\lambda} \mathrm{FA}_{\mathbb{Q}}$ (disctn) follows by transitivity using the relationships pictured in the figure. We can use this fact to conclude the following.

Lemma $5.4 \mathbf{E}(\mathbb{Q})=F A_{\mathbb{Q}}($ disctn $)$.

\section{Proof}

We will use the fact established above: $\mathbf{E}(\mathbb{Q}) \approx^{\lambda} \mathrm{FA}_{\mathbb{Q}}$ (disctn). We now show the two inclusions for the equality: $\mathbf{E}(\mathbb{Q})=\mathrm{FA}_{\mathbb{Q}}($ disctn $)$.

- $(\subseteq)$ By the fact we have $\mathbf{E}(\mathbb{Q}) \preceq^{\lambda} \mathrm{FA}_{\mathbb{Q}}$ (disctn). Using $\mathrm{D}$ and $\mathrm{N}$, we can conclude that $\lambda$ and $\lambda^{-1}$ are in $\mathrm{FA}_{\mathbb{Q}}$ (disctn). We obtain $\mathbf{E}(\mathbb{Q}) \subseteq \mathrm{FA}_{\mathbb{Q}}$ (disctn), because for any $f(\bar{x}) \in \mathbf{E}(\mathbb{Q})$, we have $f^{\lambda} \in \mathrm{FA}_{\mathbb{Q}}$ (disctn) such that $f(\bar{x})=\lambda^{-1} \circ f^{\lambda}(\lambda(\bar{x}))$, and $\lambda^{-1} \circ f^{\lambda}(\lambda(\bar{x})) \in \mathrm{FA}_{\mathbb{Q}}$ (disctn), by closure under composition.

- $(\supseteq)$ By the fact we have $\mathrm{FA}_{\mathbb{Q}}$ (disctn) $\preceq^{\lambda} \mathbf{E}(\mathbb{Q})$. We have $\lambda, \lambda^{-1} \in \mathbf{E}(\mathbb{Q})$, so by the same reasoning as in the previous inclusion we have $\mathrm{FA}_{\mathbb{Q}}($ disctn $) \subseteq \mathbf{E}(\mathbb{Q})$.

Note that the crucial place (and only place) where the previous proof would break down for $F A_{\mathbb{Q}}(\mathrm{ctn}$ ) is at the point where we claim $\lambda \in \mathrm{FA}_{\mathbb{Q}}$ (disctn); this discontinuous function cannot be in $\mathrm{FA}_{\mathbb{Q}}$ (ctn). Thus we have completed the first and easier step of the lifting, showing how to conclude $\mathbf{E}(\mathbb{Q})=$ $F A_{\mathbb{Q}}\left(\right.$ disctn) from $\mathbf{E}(\mathbb{N})=F A_{\mathbb{N}}$.

In the second step of the lifting, the main part is to show how to pass from $\mathbf{E}(\mathbb{Q})=F A_{\mathbb{Q}}($ disctn $)$ to the following theorem.

Theorem $5.5 \mathrm{E}(\mathbb{R}) \approx_{+}^{1 / \mathcal{T W}} \mathcal{L}$

Note that the theorem follows by transitivity once we have established the relationships pictured in the lower part of the figure (the approximations appearing in the figure will be proved in the next section). Note that in the figure we pass through classes with universe $\mathbb{Q}$, so transitivity alone yields the theorem only on $\mathbb{Q}$; however, since $\mathcal{L}$ and $\mathbf{E}(\mathbb{R})$ are classes of continuous functions we obtain the approximation for all of $\mathbb{R}$. As indicated in the figure, for the pairs $\mathbf{E}(\mathbb{Q})$ and $\mathbf{E}(\mathbb{R})$, and $\mathrm{FA}_{\mathbb{Q}}$ (disctn) and $\mathrm{FA}_{\mathbb{Q}}(\mathrm{ctn})$, the approximation is true only for functions with a $1 / \mathcal{T} \mathcal{W}$ modulus. Those approximations are an important and involved part of the lifting process, showing that we can in fact connect the continuous and discontinuous classes of functions simply by restricting the discontinuous classes to their functions which have a $1 / \mathcal{T W}$ modulus. Section 6 is devoted to the technical work of proving these relationships.

To obtain a class of functions which actually equals $\mathbf{E}(\mathbb{R})$ we will add a kind of limit operation to $\mathcal{L}$ (the idea of using a limit operation goes back to work from Costa and Mycka, see [9] and [10]). We will show that two different kinds of limit operations suffice and then at the end of this section 
we point out that for our purposes we could in fact be quite flexible in choosing our limit operation. We start with a limit operation that resembles LIM $_{\omega}$ (definition 8 from [1]), and, as discussed at the end of this section, is equivalent for our purposes.

Definition 5.6 $d L I M$ is the operation which takes a function function $f(t, \bar{x})$ and if $\left|\frac{\partial}{\partial t} f\right| \leq 1 / 2^{t}$ for $t \geq 1$, it returns $F(\bar{x})=\lim _{t \rightarrow \infty} f(t, \bar{x})$.

Note that the derivative condition guarantees the existence of the limit (see the end of the proof of theorem 5.8 in the next section). In [1], the function algebra $\mathcal{L}^{*}$ is defined as the function algebra $\mathcal{L}$ augmented by a limit operation (one more restrictive than $\operatorname{LIM}_{\omega}$ ). They then show (in theorem 1) that $\mathbf{E}(\mathbb{R})=\mathcal{L}^{*}$ for certain $\mathcal{C}^{2}$ functions. Instead of $\mathcal{L}^{*}$, we will consider $\mathcal{L}(\mathrm{dLIM})$, where in general by $\mathcal{F}$ (op), for a class of functions $\mathcal{F}$ and an operation op, we mean the set of functions $\mathcal{F}$ together with those that result from a single application of op to a function in $\mathcal{F}$. With this caveat and the fact that we only consider total functions on $\mathbb{R}$, we prove, in theorem 5.8 , the following, which is essentially an extension of theorem 1 from [1]:

$$
\mathbf{E}(\mathbb{R})=\mathcal{L}(\mathrm{dLIM}) .
$$

While we should be able to obtain exactly the result of [1], with a little more technical work, it seems more interesting to focus on this simple modification. In fact, we prove what they call a kind of "normal form" (as in proposition 17 of [1]), in which we only need one application of limits. Furthermore, our result does not require a restriction to $\mathcal{C}^{2}$ functions. In the conclusion of this section we discuss further reasons for choosing to focus on this simpler form. Our work will in fact use a different limit operation, one which is only concerned with closeness (again, we will discuss at the end of this section how the limit can be made quite a bit more flexible without any changes to the results).

Definition 5.7 LIM is the operation which takes a function $f(t, \bar{x})$ and returns $F(\bar{x})=\lim _{t \rightarrow \infty} f(t, \bar{x})$ if the limit exists and $F \preceq^{1 / t} f$, for $t \geq 1$.

We now state the main theorem (proved in the next section).

Theorem $5.8 \mathrm{E}(\mathbb{R})=\mathcal{L}(L I M)=\mathcal{L}(d L I M)$

To understand an important aspect of our alternative proof, consider their method for proving $\mathbf{E}(\mathbb{R})=\mathcal{L}^{*}$ (restricted to certain functions). They prove the more involved inclusion, $\mathbf{E}(\mathbb{R}) \subseteq \mathcal{L}^{*}$, by showing how to simulate Turing Machines in $\mathcal{L}^{*}$. Our proof of these results will follow without another Turing Machine simulation (i.e. nothing beyond the original one used to show $\mathbf{E}(\mathbb{N})=F A_{\mathbb{N}}$ ). As discussed, we use a lifting process which depends on showing a series of approximations. Though ultimately our proofs may not be simpler than the proofs of [1], we believe this work offers a nice perspective on these problems, as well as providing tools that should be more generally applicable.

We now discuss some of the promised issues with limits. The limits could be strengthened or weakened in various ways, preserving the same set of functions when $\mathcal{L}$ is closed under such operations. To strengthen the limits we could for instance (following the form of the limits from [1]) only require $F(\bar{x}) \preceq^{\varepsilon} f(t, \bar{x})$, where $\varepsilon(t, \bar{x})=K(\bar{x}) / \alpha(t, \bar{x})$, for some $K \in \mathcal{T} \mathcal{W}$ and $\alpha(t, \bar{x})=$ $\log \circ \ldots \circ \log (t+\bar{x})$, and we could require this only need hold for $|t| \geq s(\bar{x})$, for $s \in \mathcal{T} \mathcal{W}$. If $\mathcal{L}$ were closed under such a limit it would yield the same set of functions as closing under LIM. The reason for this is simply that given a function $f(t, \bar{x}) \in \mathcal{L}$ converging (as $t \rightarrow \infty$ ) to $F(\bar{x}$ ) 


\begin{tabular}{|c|c|c|c|c|}
\hline & $\begin{array}{l}\text { Turing } \\
\text { machine } \\
\text { models }\end{array}$ & & $\begin{array}{l}\text { Function } \\
\text { algebras }\end{array}$ & \\
\hline & $\mathbf{E}(\mathbb{N})$ & $=$ & $\mathrm{FA}_{\mathbb{N}}$ & \\
\hline \multirow[t]{3}{*}{ prop. 5.3} & $\vec{r} \mid 人$ & & $\widehat{r} \mid$ & Cor. 4.14 , Prop. 4.9 \\
\hline & $\mathbf{E}(\mathbb{Q})$ & $\approx^{\lambda}$ & $\mathrm{FA}_{\mathbb{Q}}($ disctn $)$ & \\
\hline & & $\downarrow \quad$ Lem. $\quad 5.4$ & & \\
\hline \multirow{6}{*}{ Lem. 6.7} & $\mathbf{E}(\mathbb{Q})$ & $=$ & $\mathrm{FA}_{\mathbb{Q}}($ disctn $)$ & \\
\hline & & $\leftarrow \underset{\text { if } f \text { has a } 1 / \mathcal{T W}}{\text { modulus }} \rightarrow$ & 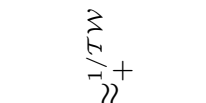 & Lem. 6.11 \\
\hline & $\vec{E}+$ & & $\mathrm{FA}_{\mathbb{Q}}(\mathrm{ctn})$ & \\
\hline & & & $\underset{2}{\frac{E}{2}}+$ & Cor. $4.25,6.14$ \\
\hline & $\mathbf{E}(\mathbb{R})$ & $\begin{array}{l}\text { Thm. } 5.5 \\
\approx_{+}^{1 / \mathcal{T} \mathcal{W}}\end{array}$ & $\mathcal{L}$ & \\
\hline & $\mathbf{E}(\mathbb{R})$ & $\begin{array}{ll}\downarrow & \text { Thm. } 5.8 \\
= & \end{array}$ & $\mathcal{L}(\mathrm{LIM})$ & \\
\hline
\end{tabular}

Figure 1: Approximations used for lifting. 
in the looser sense, we could find a function which converged in the sense of LIM, simply by taking $f(\alpha(t, \bar{x}), \bar{x}) \in \mathcal{L}$ for $\alpha(t, \bar{x})$ sufficiently fast. We could even allow repeated use of limits (though not allowing other operations after a limit). To weaken the limit we could define a kind of completion operation, putting $F(\bar{x})$ in the completion of $\mathcal{L}$ if for every $\alpha(t, \bar{x}) \in \mathcal{T} \mathcal{W}$, there were a $f(t, \bar{x}) \in \mathcal{L}$ such that $F \preceq^{1 / \alpha} f$ (in which case $\lim _{t \rightarrow \infty} f(t, \bar{x})=F(\bar{x})$ ). This is again equivalent to LIM, by using the same idea of composing in fast growing functions. For dLIM the situation is similar with the caveat that the function bounding the derivative, say $b(t, \bar{x})$, needs to converge to zero quickly enough as $t \rightarrow \infty$ (basically a condition like $\sum_{t \in \mathbb{N}} b(t, \bar{x})<\infty$ would suffice, and using $b(t, \bar{x})=1 / 2^{t}$ is a simple approach; this point follows by inspecting the end of the proof of theorem 5.8 in the next section). Furthermore, for dLIM, we can not simply compose in fast functions because the derivative would not be properly bounded, but we can use lemma 6.2, which allows us to transform a converging function (which could have a badly behaved derivative) into a function that converges with a properly bounded derivative. Note in particular that we can obtain the claim $\mathbf{E}(\mathbb{R})=\mathcal{L}\left(\mathrm{LIM}_{\omega}\right)$, essentially theorem 1 of [1].

This latter idea of completion highlights what is really going on. The fundamental point is that $\mathcal{L}$ in some sense approximates $\mathbf{E}(\mathbb{R})$ (e.g. theorem 5.5), so that any sufficiently well behaved way of completing it yields $\mathbf{E}(\mathbb{R})$. As seen in the above discussion the manner in which $\mathcal{L}$ can be completed is quite flexible. The function algebra $\mathcal{L}^{*}$ of [1] is a good first step towards finding a function algebra for $\mathbf{E}(\mathbb{R})$, but in a sense it is not a "proper" function algebra. Their limit operation can be interleaved with the other operations of the function algebra as one would like, but they go on to prove a "normalization" theorem, showing that a single use of the limit operation suffices. In a more "proper" function algebra we would expect the necessary interleaving of the operations in order to obtain all the functions of the class. From this discussion we justify our ignoring the issue of showing how the limit operation can be used freely in the function algebra. However the initial step of [1] leads to an interesting, though not precisely stated question.

Question 5.9 Are there natural and genuine operations that can be added to the function algebra $\mathcal{L}$ (or perhaps to a different function algebra) so that the resulting functions are exactly $\mathbf{E}(\mathbb{R})$, where by "genuine" we mean that it really makes sense to interleave these operations with the other operations of the function algebra?

We remark that the idea of completion is at the core of the notion of "converging computation" for continuous-time models like the GPAC (or, equivalently, systems of polynomial differential equations) explored in [5] and [11]. The main result in [11] could be stated as an approximation result for the real computable functions. The equivalence proved in that paper between GPACcomputability and computability in the sense of recursive analysis follows from the fact that the definition of GPAC-computability encapsulates the idea of completion. The connections above suggest that approximation, lifting and completion should allow us to unify apparently distinct notions of computability over the reals.

\section{Connection to Computable Analysis: Technical Discussion}

The rest of the paper is devoted to proving the technical lemmas and the properties indicated in the figure of the previous section, as well as providing a proof of theorem 5.8.

See [3] for the details on the following technical lemma (in particular, see lemma 4.6 and 4.7 of [3] for a discussion of the functions behaving like $\sigma$ and step). 
Lemma 6.1 $\mathcal{L}$ contains the functions,$+ *$, and $\sin$, as well as functions step and $\sigma$ with the following properties:

- $\sigma(t)=0$ for $t \leq 0$ and $\sigma(t)=1$, for $t \geq 1$.

- $\operatorname{step}(t)=\lfloor t\rfloor$ if $t \in[\lfloor t\rfloor,\lfloor t\rfloor+1 / 2]$, and step is increasing.

Thus we will freely use such functions in $\mathcal{L}$, using the names $\sigma$ and step to refer to functions in $\mathcal{L}$ with the above properties.

The next lemma shows how to transform a converging function of $\mathcal{L}$ into one that converges with a well-behaved derivative.

Lemma 6.2 Suppose $f(t, \bar{x}) \in \mathcal{L}$ such that $\lim _{t \rightarrow \infty} f(t, \bar{x})=F(\bar{x})$ and $F \preceq^{1 / t} f$. For any $\alpha(t, \bar{x}) \in$ $\mathcal{T} \mathcal{W}$ there is $h(t, \bar{x}) \in \mathcal{L}$ such that $\lim _{t \rightarrow \infty} h(t, \bar{x})=F(\bar{x})$ and $\left|\frac{\partial}{\partial t} h\right| \leq 1 / \alpha$.

\section{Proof}

Let $\alpha^{*}(t, \bar{x})$ be a sufficiently fast function in $\mathcal{L}$ (discussed later). Let $g(t, \bar{x})=f\left(\alpha^{*}(t, \bar{x}), \bar{x}\right)$, so $F \preceq^{1 / \alpha^{*}} g$. Let $G(t, \bar{x})=g(\operatorname{step}(t), \bar{x})$, so $G(t, \bar{x})=g(\lfloor t\rfloor, \bar{x})$ for $t \in[\lfloor t\rfloor,\lfloor t\rfloor+1 / 2]$. Let $H(t, \bar{x})=\sigma(2 t+1 / 2) c_{3} \theta_{3}(\sin 2 \pi t) G(t, \bar{x})$, where $c_{3}$ is a constant we can define in $\mathcal{L}$, which makes $\int_{0}^{1 / 2} c_{3} \theta_{3}(\sin 2 \pi t)=1$ (see proofs of lemma 4.6 and 4.7 of [3]). The particularly relevant properties of $H$ are as follows.

1. $H(t, \bar{x})=0$ for $-1 \leq t \leq 0$ (this property is the only reason for the function $\sigma(2 t+1 / 2)$, which equals 1 for $t \geq 0$ and so we mostly ignore).

2. $H(t, \bar{x})=0$ for $t \in[\lfloor t\rfloor+1 / 2,\lfloor t\rfloor+1]$ for $t \geq 0$.

3. $\int_{\lfloor t\rfloor}^{\lfloor t\rfloor+1 / 2} H(u, \bar{x}) d u=g(\lfloor t\rfloor, \bar{x})$, for $t \geq 0$.

Let $h(t, \bar{x})=\int_{0}^{t} H(u, \bar{x})-H(u-1, \bar{x}) \mathrm{d} u$, which is in $\mathcal{L}$ because $H$ is, and integration is a special case of $\mathbf{L I}$.

Now we check that $h$ works as required. For the derivative condition consider $t \geq 0$ and note that:

$$
\begin{aligned}
\left|\frac{\partial}{\partial t} h\right| & =|H(t, \bar{x})-H(t-1, \bar{x})| \\
& \leq c_{3}|G(t, \bar{x})-G(t-1, \bar{x})|
\end{aligned}
$$

By the definition of $G$ and the fact that the function step is monotone, the last difference can be bounded by $\max _{\lfloor t\rfloor-1 \leq t_{1}, t_{2} \leq\lfloor t\rfloor+1}\left\{c_{3}\left|g\left(t_{1}, \bar{x}\right)-g\left(t_{2}, \bar{x}\right)\right|\right\}$, so by choosing $\alpha^{*}$ sufficiently large (around $2 \alpha$ ), since $F \preceq^{1 / \alpha^{*}} g$, we can get $\left|\frac{\partial}{\partial t} h\right| \leq 1 / \alpha$.

To show that $\lim _{t \rightarrow \infty} h(t, \bar{x})=F(\bar{x})$, it suffices to show that $h(t, \bar{x})$ is in-between $g(\lfloor t\rfloor-$ 
$1, \bar{x})$ and $g(\lfloor t\rfloor, \bar{x})$, for $t \geq 1$. Note that (leaving out $\bar{x}$, and for $t \geq 1$ ):

$$
\begin{aligned}
h(t) & =\int_{0}^{t} H(u)-H(u-1) \\
& =\int_{t-1}^{t} H(u) \\
& =\int_{\lfloor t\rfloor-1}^{\lfloor t\rfloor} H(u)-\int_{\lfloor t\rfloor-1}^{t-1} H(u)+\int_{\lfloor t\rfloor}^{t} H(u) \\
& =g(\lfloor t\rfloor-1, \bar{x})-g(\lfloor t\rfloor-1, \bar{x}) \int_{\lfloor t\rfloor-1}^{t-1} c_{3} \theta_{3}(\sin 2 \pi u)+g(\lfloor t\rfloor, \bar{x}) \int_{\lfloor t\rfloor}^{t} c_{3} \theta_{3}(\sin 2 \pi u) \\
& =(1-\gamma(t)) g(\lfloor t\rfloor-1, \bar{x})+\gamma(t) g(\lfloor t\rfloor, \bar{x}),
\end{aligned}
$$

where $\gamma(t)=\int_{\lfloor t\rfloor-1}^{t-1} c_{3} \theta_{3}(\sin 2 \pi u)=\int_{\lfloor t\rfloor}^{t} c_{3} \theta_{3}(\sin 2 \pi u)$. Thus $h(t, \bar{x})$ is in-between $g(\lfloor t\rfloor-$ $1, \bar{x})$ and $g(\lfloor t\rfloor, \bar{x})$ as desired. For the second equality, we use property 1 of $H$, along with basic properties of integrals. The second to last equality follows by the definition of $G$ along with properties 2 and 3 of $H$.

Now we prove the main theorem (theorem 5.8) from the last section:

$$
\mathbf{E}(\mathbb{R})=\mathcal{L}(\mathrm{LIM})=\mathcal{L}(\mathrm{dLIM})
$$

\section{Proof}

We will use the following fact:

$$
\mathbf{E}(\mathbb{R})(\operatorname{LIM})=\mathbf{E}(\mathbb{R})
$$

First we prove this fact. Suppose $f(t, \bar{x}) \in \mathbf{E}(\mathbb{R})$ and $F(\bar{x})=\lim _{t \rightarrow \infty} f(t, \bar{x})$, with $F \preceq^{1 / t} f$. We need to show $F \in \mathbf{E}(\mathbb{R})$. Let $M^{\bar{x}, t}(n)$ be the machine that approximates $f(t, \bar{x})$ to $1 / n$ accuracy, writing the oracle inputs $\bar{x}, t$ as superscripts. Let $N^{\bar{x}}(n)$ be defined by $M^{\bar{x}, 2 n}(2 n)$, using accuracy input $2 \mathrm{n}$ as the oracle input for $t$. Thus $\mid f(2 n, \bar{x})-$ $N^{\bar{x}}(n) \mid \leq 1 / 2 n$ and $|F(\bar{x})-f(2 n, \bar{x})| \leq 1 / 2 n$, so $\left|F(\bar{x})-N^{\bar{x}}(n)\right| \leq 1 / n$, and $F \in \mathbf{E}(\mathbb{R})$.

Now consider the first equality $\mathbf{E}(\mathbb{R})=\mathcal{L}($ LIM $)$. By theorem 5.5 we have the approximation $\mathbf{E}(\mathbb{R}) \approx_{+}^{1 / \mathcal{T} \mathcal{W}} \mathcal{L}$, which we use to show both inclusions of the first equality.

- $\subseteq$ : Consider $f(\bar{x}) \in \mathbf{E}(\mathbb{R})$ and let $f^{*}(t, \bar{x}) \in \mathcal{L}$ such that $f \preceq^{1 / t} f^{*}$. Thus $f(\bar{x})=$ $\lim _{t \rightarrow \infty} f^{*}(t, \bar{x}) \in \mathcal{L}(\mathrm{LIM})$.

- $\supseteq$ : Consider $f(\bar{x}) \in \mathcal{L}$ and let $f^{*}(t, \bar{x}) \in \mathbf{E}(\mathbb{R})$ such that $f \preceq^{1 / t} f^{*}$. Thus $f(\bar{x})=$ $\lim _{t \rightarrow \infty} f^{*}(t, \bar{x}) \in \mathbf{E}(\mathbb{R})(\mathrm{LIM})=\mathbf{E}(\mathbb{R})$, the last equality holding by the above fact. Thus $\mathcal{L} \subseteq \mathbf{E}(\mathbb{R})$, and so $\mathcal{L}(\mathrm{LIM}) \subseteq \mathbf{E}(\mathbb{R})(\mathrm{LIM})=\mathbf{E}(\mathbb{R})$.

Consider the second equality $\mathcal{L}(\mathrm{LIM})=\mathcal{L}(\mathrm{dLIM})$. We show both inclusions.

- $\subseteq$ : Suppose $F(\bar{x})=\lim _{t \rightarrow \infty} f(t, \bar{x})$ for $f \in \mathcal{L}$, and $F \preceq^{1 / t} f$. To show $F \in \mathcal{L}(\mathrm{dLIM})$ we need an $h(u, \bar{x}) \in \mathcal{L}$ such that $\lim _{u \rightarrow \infty} h(u, \bar{x})=F(\bar{x})$ and $\left|\frac{\partial}{\partial u} h\right| \leq 1 / 2^{u}$; such an $h$ is given by lemma 6.2 . 
- $\supseteq$ : Suppose $f(t, \bar{x})$ is such that $\left|\frac{\partial}{\partial t} f\right| \leq 1 / 2^{t}$. From the derivative condition we can conclude that $\left|f(t, \bar{x})-f\left(t_{0}, \bar{x}\right)\right| \leq 1 / 2^{t_{0}}$, for $t_{0} \leq t \leq t_{0}+1$, and thus

$$
\star\left|f(t, \bar{x})-f\left(t_{0}, \bar{x}\right)\right| \leq 1 / 2^{t_{0}}+1 / 2^{t_{0}+1}+\ldots \leq 1 / 2^{t_{0}-1} \text {, for } t \geq t_{0} .
$$

Thus, for fixed $\bar{x}, \lim _{t \rightarrow \infty} f(t, \bar{x})$ exists and we call this limit $F(\bar{x})$. To finish, it suffices to show that $|F(\bar{x})-f(t, \bar{x})| \leq 1 / 2^{t-2}$ (which is $\leq 1 / t$ for $t \geq 4$ ). To show this, assume for sake of contradiction that there are $t_{0}, \bar{x}$ such that $|F(\bar{x})-f(t, \bar{x})|>$ $1 / 2^{t_{0}-2}$. For ease of discussion, assume $f\left(t_{0}, \bar{x}\right)<F(\bar{x})$. By condition $\star$, for $t>t_{0}$,

$$
f(t, \bar{x}) \leq f\left(t_{0}, \bar{x}\right)+1 / 2^{t_{0}-1} \leq F(\bar{x})-1 / 2^{t_{0}-1},
$$

which contradicts the fact that $\lim _{t \rightarrow \infty} f(t, \bar{x})=F(\bar{x})$.

Definition 6.3 For functions $f(x, \bar{y})$ and $g(x, \bar{y})$, and $a \in \mathbb{Q}$ such that $f(a, \bar{y})=g(a, \bar{y})$, we define the operation $h(x, \bar{y})=$ merge $_{a}(f, g)$ so that $h(x, \bar{y})= \begin{cases}f(x, \bar{y}), & \text { if } x \leq a \text {; } \\ g(x, \bar{y}), & \text { if } x>a .\end{cases}$

Lemma 6.4 Let $\mathcal{F}$ be the set of $\mathbb{R}$-functions with a $1 / \mathcal{T} \mathcal{W}$-modulus. For any $a \in Q$,

$$
\left(\text { merge }_{a}\right)_{\mid \mathcal{F}} \preceq_{+}^{1 / \mathcal{T W}} \mathcal{L}
$$

\section{Proof}

Let $f(x, \bar{y})$ and $g(x, \bar{y})$ be functions with a $1 / \mathcal{T} \mathcal{W}$-modulus, such that $f(a, \bar{y})=g(a, \bar{y})$, and let $h(x, \bar{y})=\operatorname{merge}_{a}(f, g)$. Supposing $f^{*}(x, \bar{y}, \bar{z})$ and $g^{*}(x, \bar{y}, \bar{z}) 1 / \mathcal{T} \mathcal{W}$ approximate $f$ and $g$, respectively, we define $h^{*}(x, \bar{y}, \bar{z})$ that $1 / \mathcal{T} \mathcal{W}$ approximates $h$. We define $h^{*}$ by a convex combination of $f^{*}$ and $g^{*}$ given by $h^{*}(x, \bar{y}, \bar{z})=\left(1-c_{a}(x, \bar{y}, \bar{z})\right) f^{*}(x, \bar{y}, \bar{z})+$ $c_{a}(x, \bar{y}, \bar{z}) g^{*}(x, \bar{y}, \bar{z})$, where $c_{a}(x, \bar{y}, \bar{z})=0$ for $x \leq a, c_{a}(x, \bar{y}, \bar{z})=1$ for $x \geq a^{\prime}>a$ and $c_{a}$ increases with $x$ on $\left[a, a^{\prime}\right]$, where $a^{\prime}$ will be defined so it is just a bit bigger than $a$. The idea is to define $c_{a}$ in $\mathcal{L}$ such that $h \preceq_{+}^{1 / \mathcal{T} \mathcal{W}} h^{*}$.

We define $c_{a}(x, \bar{y}, \bar{z})=\sigma\left(M(x, \bar{y}, \bar{z}) \theta_{3}(x-a)\right)$, where $M$ is a fast growing function in $\mathcal{T W}$, which we easily dominate in $\mathcal{L}$. It is easy to check that $c_{a}$ behaves as indicated above and, therefore $h^{*}=f^{*}$ or $h^{*}=g^{*}$ for $x \leq a$ and $x \geq a^{\prime}$, respectively. Moreover, we can adjust $\left|a^{\prime}-a\right|$ with $M$ so that $h^{*}$ works on $\left[a, a^{\prime}\right]$ too. To see why, first note that by the definition of $c_{a}$, for $x \in\left[a, a^{\prime}\right]$ we have that $h^{*}(x, \bar{y}, \bar{z})$ is between $f^{*}(a, \bar{y}, \bar{z})$ and $g^{*}\left(a^{\prime}, \bar{y}, \bar{z}\right)$. We can bound $\left|f^{*}(a, \bar{y}, \bar{z})-g^{*}\left(a^{\prime}, \bar{y}, \bar{z}\right)\right|$ freely in $1 / \mathcal{T} \mathcal{W}$, because we can freely bound $\left|f(a, \bar{y})-f^{*}(a, \bar{y}, \bar{z})\right|,\left|g\left(a^{\prime}, \bar{y}\right)-g^{*}\left(a^{\prime}, \bar{y}, \bar{z}\right)\right|$, and $\left|f(a, \bar{y})-g\left(a^{\prime}, \bar{y}\right)\right|$, the latter bound following from the modulus condition on $g$.

The next important technical lemma shows that we can approximate linearizations.

Lemma 6.5 $\operatorname{Lin} \preceq_{+}^{1 / \mathcal{T} \mathcal{W}} \mathcal{L}$

Proof 
Suppose $f(x, \bar{y})$ is some $\mathbb{R}$-function and $\alpha(x, \bar{y}, \bar{z}) \in \mathcal{T} \mathcal{W}$, and let $L(x, \bar{y})=\operatorname{Lin}(f(x, \bar{y}) ; x)$ (linearizing with respect to more variables follows). We will describe an $L^{*}(x, \bar{y}, \bar{z})$ such that $L \preceq^{1 / \alpha} L^{*}$, using $f^{*}(x, \bar{y}, \bar{z})$ such that $f \preceq^{1 / \beta} f^{*}$ and the extra function $u(x, \bar{z})$, where $\beta(x, \bar{y}, \bar{z}) \in \mathcal{T} \mathcal{W}$; at the end both $u$ and $\beta$ will be chosen large enough in $\mathcal{T} \mathcal{W}$ to get the $1 / \alpha$ approximation.

For simplicity we drop the argument $\bar{y}$ in the proof and we consider $x \geq 0$. Consider the piecewise constant function $\Delta(x)=f(\lfloor x\rfloor+1)-f(\lfloor x\rfloor)$ which is the slope of $L(x)$ for non integer $x . \Delta$ is discontinuous, so instead we develop a continuous "step" function $S$ which will behave like $\Delta(x)$. This is done in three steps. Firstly, we define with linear integration a pair of functions $y_{1}, y_{2}$ that approximate $f^{*}(\lfloor x\rfloor)$ and $f^{*}(\lfloor x\rfloor-1)$, respectively on $\left[\lfloor x\rfloor+\frac{1}{2},\lfloor x\rfloor+1\right]$ and $\left[\lfloor x\rfloor,\lfloor x\rfloor+\frac{1}{2}\right]$, respectively. Then, we use $y_{1}, y_{2}$ to define new functions $S_{1}$ and $S_{2}$ that similarly approximate $\Delta(\lfloor x\rfloor)$. Finally, $S$ is given by a convex combination of $S_{1}$ and $S_{2}$. Having $S$, we define $h$ as the solution of $\frac{\partial}{\partial x} h(x, u)=S(x, u)$ with initial condition $h(0, u)=f^{*}(0)$, where the new parameter $u$ controls the error of the approximation. Finally, a function $L^{*}$ such that $L \preceq^{1 / \alpha} L^{*}$ is given by $L^{*}(x, \bar{z})=h(x, u(x, \bar{z}))$ where $u$ is an appropriate increasing function of $x$ and $\bar{z}$.

Then $F(x)=f^{*}(\operatorname{step}(x))=f^{*}(\lfloor x\rfloor)$ for $x \in\left[\lfloor x\rfloor,\lfloor x\rfloor+\frac{1}{2}\right]$. We define $y_{1}, y_{2}$ with the following system of linear differential equations

$$
\begin{aligned}
& \frac{\partial}{\partial x} y_{1}(x, u)=\left(F(x)-y_{1}\right) \theta_{3}(\sin 2 \pi x) u \\
& \frac{\partial}{\partial x} y_{2}(x, u)=\left(y_{1}-y_{2}\right) \theta_{3}(-\sin 2 \pi x) u
\end{aligned}
$$

and initial condition $y_{1}(0, u)=y_{2}(0, u)=0$. The equations above can be explicitly solved by separating variables. For $x \in\left[\lfloor x\rfloor+\frac{1}{2},\lfloor x\rfloor+1\right]$, the error $\left|y_{1}(x, u)-f^{*}(\lfloor x\rfloor)\right|$ is bounded by

$$
e(\lfloor x\rfloor, u)=\exp \left\{-u \int_{\lfloor x\rfloor}^{\lfloor x\rfloor+1 / 2} \sin ^{3}(2 \pi t) d t\right\}
$$

This can be freely bounded in $1 / \mathcal{T} \mathcal{W}$ adjusting $u$. It is easy to check that $y_{2}$ behaves similarly, with a $1 / 2$ delay. Hence, $S_{1}(x, u)=y_{2}(x+2, u)-y_{2}(x+1, u)$ and $S_{2}(x, u)=$ $y_{1}(x+1, u)-y_{1}(x, u)$ approximate $\Delta(\lfloor x\rfloor)$ respectively on $\left[\lfloor x\rfloor,\lfloor x\rfloor+\frac{1}{2}\right]$ and $[\lfloor x\rfloor+$ $\left.\frac{1}{2},\lfloor x\rfloor+1\right]$ with an error bounded by $2 e(\lfloor x\rfloor, u)$.

Consider now the convex combination $S(x, u)=\lambda(x, u) S_{1}(x, u)+(1-\lambda(x, u)) S_{2}(x, u)$, where $\lambda(x, u)=\sigma\left(u \theta_{3}(\sin 2 \pi x)\right)$ is a function with period 1 whose values range over $[0,1]$. More precisely, there is a function $\epsilon_{u}$ which decreases to zero as $u$ increases, such that $\lambda$ can be described by its behavior on the following intervals $(n \in \mathbb{N})$, which essentially break up each interval $[n, n+1]$ into its left half $I_{\mathrm{L}}$, its right half $I_{\mathrm{R}}$, and two small pieces:

$$
\begin{aligned}
& I_{\mathrm{L}}^{n}=\left[n+\epsilon_{u}, n+\frac{1}{2}-\epsilon_{u}\right], \quad I_{\epsilon}^{n}=\left[n-\epsilon_{u}, n+\epsilon_{u}\right], \\
& I_{\mathrm{R}}^{n}=\left[n+\frac{1}{2}+\epsilon_{u}, n+1-\epsilon_{u}\right], \quad J_{\epsilon}^{n}=\left[n+\frac{1}{2}-\epsilon_{u}, n+\frac{1}{2}+\epsilon_{u}\right] .
\end{aligned}
$$

We have that $\lambda(x, u)=1$ for $x \in I_{\mathrm{L}}^{\lfloor x\rfloor}, \lambda(x, u)=0$ for $x \in I_{\mathrm{R}}^{\lfloor x\rfloor}$, and $\lambda$ is monotone on $I_{\epsilon}^{\lfloor x\rfloor}$ and on $J_{\epsilon}^{\lfloor x\rfloor}$. 
Given $S$, we define $h(x, u)=f^{*}(0)+\int_{0}^{x} S(t, u) d t$. The total error $|h(x, u)-L(x)|$ can be broken in two parts, one on $X=\cup_{n=0}^{\lceil x\rceil}\left(I_{\mathrm{L}}^{n} \cup I_{\mathrm{R}}^{n}\right)$, and the other one on $X_{\epsilon}=\cup_{n=0}^{\lceil x\rceil}\left(I_{\epsilon}^{n} \cup J_{\epsilon}^{n}\right)$. Then,

$$
\begin{aligned}
|h(x, u)-L(x)| & \leq \int_{X} S(x, u)-\Delta(x) d x+\int_{X_{\epsilon}} S(x, u)-\Delta(x) d x \\
& <\Sigma_{n=0}^{\lceil x\rceil} 2 e(n, u)(2 / \beta(n))+\Sigma_{n=0}^{\lceil x\rceil} \epsilon_{u} \Delta(n)(2 / \beta(n))
\end{aligned}
$$

The $(2 / \beta(n))$ terms appear because $S$ in fact approximates $f^{*}(\lfloor x\rfloor+1)-f^{*}(\lfloor x\rfloor)$, which approximates $\Delta(x)=f(\lfloor x\rfloor+1)-f(\lfloor x\rfloor)$ to $2 / \beta(\lfloor x\rfloor)$ accuracy. Both terms decrease with $u$ and $\beta$. In particular, the second term can be bounded by choosing $\beta$ sufficiently large and $u(x, \bar{z})$ sufficiently large to make $\epsilon_{u}$ sufficiently small in $1 / \mathcal{T} \mathcal{W}$. Hence, there is are large enough $u(x, \bar{z})$ in $\mathcal{L}$ and $\beta \in \mathcal{T} \mathcal{W}$ such that we can freely bound $|h(x, u(x, \bar{z}))-L(x)|$ in $1 / \mathcal{T} \mathcal{W}$. Therefore, $L^{*}(x, \bar{z})=h(x, u(x, \bar{z}))$ satisfies $L \preceq^{1 / \alpha} L^{*}$.

The next proposition follows by a small calculation; we write $[u]$ in a statement to indicate that it holds for either $\lfloor u\rfloor$ or $\lceil u\rceil$ in place of $[u]$.

Proposition 6.6 Given $r(x, \bar{w}) \in \mathcal{T} \mathcal{W}$ there is $r^{*}(x, \bar{w}) \in \mathcal{T} \mathcal{W}$ such that for any $R(x, \bar{w}) \geq$ $r^{*}(x, \bar{w})$, we have

$$
\left|x-\frac{[R(x, \bar{w}) x]}{[R(x, \bar{w})]}\right| \leq \frac{1}{r(x, \bar{w})} .
$$

The following lemma makes the basic connection between the two models of computation on Turing Machines, the Computable Analysis model and the discrete model.

Lemma 6.7 $\mathbf{E}(\mathbb{R}) \approx_{+}^{1 / \mathcal{T} \mathcal{W}}\{f \in \mathbf{E}(\mathbb{Q}) \mid f$ has a modulus in $1 / \mathcal{T} \mathcal{W}\}$.

\section{Proof}

We prove the two approximate inclusions.

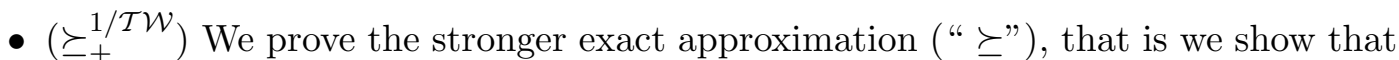
for $f(x) \in \mathbf{E}(\mathbb{Q})$ (we consider just one variable for simplicity), with a modulus $1 / m(x, z) \in 1 / \mathcal{T} \mathcal{W}$, there is $f^{*}(x) \in \mathbf{E}(\mathbb{R})$ such that $f(x)=f^{*}(x)$ for $x \in \mathbb{Q}$. Let $M$ be the Turing Machine which computes $f$ where the input $x \in Q$ is given as a triple of natural numbers. Let $f^{*}$ be the unique continuous extension of $f$ to $\mathbb{R}$.

We design a Turing Machine $N$ to put $f^{*}$ in $\mathbf{E}(\mathbb{R}) . N$ has an oracle for $x$ and an accuracy input $z$. First $N$ computes $m\left(x^{*}, z\right)$, where $x^{*}$ is a number such that $x \leq x^{*}$ (easily obtained by querying for close enough approximation to $x$ and then adding one). $N$ puts $m\left(x^{*}, z\right)$ on the query tape to get some $y \in \mathbb{D}$ such that $|x-y| \leq 1 / m\left(x^{*}, z\right) \leq 1 / m(x, z)$ (the last inequality holds since functions in error classes decrease). Also note that $y$ is a dyadic of length $\leq m\left(x^{*}, z\right)$ (by usual definitions in Computable Analysis, see [7], definition 2.1, requirement "prec $(\phi(n))=n ")$ and so for $y=p / q$, we have $p, q \leq m\left(x^{*}, z\right)$. Now $N$ simply runs $M$ on $(p, q)$, thus outputing exactly $f(y)$ (we ignore the sign of $y$ for simplicity), and due to the modulus condition we know that $|f(x)-f(y)| \leq 1 / z$. $N$ runs within $\mathcal{T W}$ time in $x$ and $z$ because the length of the $p$ and $q$ are larger by at most a function in $\mathcal{T W}$ and M's running time is bounded by a function in $\mathcal{T} \mathcal{W}$. 
- $\left(\swarrow_{+}^{1 / \mathcal{T} \mathcal{W}}\right)$ Let $f(\bar{x}) \in \mathbf{E}(\mathbb{R})$, and let $\alpha(\bar{x}, \bar{y}) \in \mathcal{T} \mathcal{W}$, and we need $f^{*}(\bar{x}, \bar{y}) \in \mathbf{E}(\mathbb{Q})$ such that $f \preceq^{1 / \alpha} f^{*}$ and $f^{*}$ has a modulus in $1 / \mathcal{T} \mathcal{W}$. Let $M$ be the Turing Machine that computes $f$ in the Computable Analysis sense of approximation. Thus $M$ has an oracle tape which gives approximations of $\bar{x}$, and an input tape for the accuracy input. We will design a Turing Machine $N$ which takes $\bar{x}, \bar{y} \in \mathbb{Q}$ as input (where each rational is given exactly as a triple of natural numbers); $f^{*}(\bar{x}, \bar{y})$ will be the function computed by $N$. To obtain the condition $f \preceq^{1 / \alpha} f^{*}$ alone would be straightforward. We could define $N$ in terms of $M$, by inputing the the desired accuracy, $\lceil\alpha(\bar{x}, \bar{y})\rceil$, to the machine $M$, and use the exact $\bar{x}$ as the oracle to $M$. This is roughly how $N$ will in fact be defined, but guaranteeing the modulus condition will require some care and is the reason for complicating the definition of $N$. For ease of exposition, suppose the inputs (to machine $N$ ) $\bar{x}, \bar{y}$ are both of length 1 , so we write them as $x$ and $y$. Let $t(x, n) \in \mathcal{T} \mathcal{W}$ be the largest dyadic number that can be written down by $M$ given oracle and accuracy inputs of $x$ and $n$, respectively (i.e. about 2 raised to the time bound on the machine).

First we compute $\alpha, t \in \mathcal{T} \mathcal{W}$ exactly, since by definition they are just linearizations of functions that we can compute on $\mathbb{N}$. We also calculate $\tau(x, y)=t(x, 1+\alpha(x, y))$, and let (by proposition 6.6) $\tau^{*}(x, \bar{y}) \in \mathcal{T} \mathcal{W}$ be sufficiently large so that the 4 rationals of the form $\left[\tau^{*} x\right] /\left[\tau^{*}\right]$ are all within $1 / \tau$ of $x$. Now we will define a function $h\left(u_{1}, u_{2}, u_{3}\right)$ on $\mathbb{N}$, taking on values in $\mathbb{Q}$ :

Run $M$ with accuracy input $u_{1}$, using $u_{2} / u_{3}$ as its oracle. When we say to use $u_{3} / u_{2}$ as the oracle we mean that we consider the binary expansion of $u_{3} / u_{2}$ and whenever some accuracy is asked of the oracle, exactly enough bits of this expansion are given. Define $h\left(u_{1}, u_{2}, u_{3}\right)$ to be the output of this run of $M$.

Consider the linearization $\hat{h}\left(u_{1}, u_{2}, u_{3}\right)$ of $h$ (recall definition 2.8). We define $N(x, y)=\hat{h}\left(1+\alpha(x, y), x \tau^{*}(x, y), \tau^{*}(x, y)\right)$. It is continuous because it is the result of composing continuous functions. Furthermore this function has a $1 / \mathcal{T} \mathcal{W}$ modulus because it is the result of composing functions that have both a $1 / \mathcal{T} \mathcal{W}$ modulus and $\mathcal{T W}$ bounds (this holds by reasoning similar to that in the proof of lemma 3.13). It is left to check that it operates as required, that is that $|f(x)-N(x, y)| \leq 1 / \alpha(x, y)$. By proposition 2.9 , it suffices to note that all of the $2^{3}$ versions of $h$ (used in the definition of $N$ ) on $\mathbb{Z}$ are within $1 / \alpha(x, y)$ of $f(x)$. This is true because $h$ runs $M$, and the following two points hold concerning this run, following the definition of $N$. First, $M$ is given a sufficiently large accuracy parameter $1+\alpha$, i.e. both $\lfloor 1+\alpha\rfloor$ and $\lceil 1+\alpha\rceil$ are at least as big as $\alpha$. Second, $M$ uses an oracle for $x$ that is good enough, meaning that whenever $M$ asks for $x$ to some accuracy, it gets something that is that accurate. Since $M$ cannot write down numbers larger than $\tau$, it can only demand $x$ to an accuracy at best $1 / \tau$. By the the definition of $h$, we picked a number as the oracle that was this close to $x$ (i.e. any of the four $\left[\tau^{*} x\right] /\left[\tau^{*}\right]$ ).

To relate $\mathrm{FA}_{\mathbb{Q}}$ (disctn) and $\mathrm{FA}_{\mathbb{Q}}$ (ctn) will use some technical lemmas. The next important techni- 
cal lemma relates to the syntactic structure of the function algebra (i.e. recall the construction trees of definition 3.5) and is sensitive to the exact definition of the function algebra (i.e. other function algebras which yield the same functions in the end, might have the wrong syntactic property).

Lemma 6.8 For every $f(\bar{x}) \in F A_{\mathbb{Q}}$ (disctn), there is a construction tree for $f$ in which $D$ is only applied to variables.

\section{Proof}

It suffices to show we can push D into any of the basic functions and past any of the operations of $\mathrm{FA}_{\mathbb{Q}}$ (disctn). First we discuss some useful functions. Recall the gcd function on $\mathbb{N}$ and also consider the function $\lfloor x / y\rfloor$ on $\mathbb{N}$, where we let $\lfloor x / 0\rfloor=0$ for any $x \in \mathbb{N}$; we will use these functions on $\mathbb{Z}$, requiring gcd to always be nonnegative. By proposition 4.9, $\mathrm{FA}_{\mathbb{Q}}$ (ctn) contains extensions of these functions to $\mathbb{Q}$ (we use the same names for the extensions, only caring about their behavior on $\mathbb{Z}$ ). We also consider the two discontinuous functions, $\mathrm{N}$ and sign (recall definition 5.1). We can define sign in $\mathrm{FA}_{\mathbb{Q}}$ (disctn) in such a way that $\mathrm{D}$ is only applied to variables, as follows: $\operatorname{sign}(x)=\operatorname{sgn}\left(\theta_{1}(\mathrm{D}(x))\right)$. Likewise, $\mathrm{N}(x)=|x| \mathrm{D}(x)$. We now show how to push $\mathrm{D}$ past the basic functions:

1. $\mathrm{D}\left(\theta_{1}(x)\right)=\theta_{1}(\mathrm{D}(x))$

2. $\mathrm{D}(\mathrm{D}(x))=\left\{\begin{array}{ll}0, & \text { if } x=0 ; \\ 1, & \text { if } x>0 ; \\ -1, & \text { if } x<0 .\end{array}=\operatorname{sign}(x)-\operatorname{sign}(-x)\right.$

3. $\mathrm{D}(\operatorname{div}(x))=\left\{\begin{array}{ll}x, & \text { if } x \geq 1 ; \\ 1, & \text { if } x<1 .\end{array}=1+\theta_{1}(x-1)\right.$

4.

$$
\mathrm{D}(x y)=\left\lfloor\frac{\mathrm{D}(x) \mathrm{D}(y)}{\operatorname{gcd}(\mathrm{D}(x) \mathrm{D}(y), \mathrm{N}(x) \mathrm{N}(y))}\right\rfloor
$$

We can check this by letting $x=p / q$ and $y=a / b$ and checking the equation: $\mathrm{D}((p / q)(a / b))=\mathrm{D}(p a / b q)=b q / \operatorname{gcd}(b q, p a)$. Furthermore, the equation works for either $x$ or $y$ equals 0 (by the above definition of $\lfloor\cdot / \cdot\rfloor$ ), and the sign matches (i.e. the sign of $\mathrm{D}(x y)$ is the same as the sign of $\mathrm{D}(x) \mathrm{D}(y)$ and by definition gcd is non-negative).

5. For + we have cases on whether or not $x$ or $y$ is 0 , and use a function, say $\mathrm{s}(x, y)$, built up from sign, to make the sign correct:

$$
\mathrm{D}(x+y)= \begin{cases}\left\lfloor\frac{\mathrm{s}(x, y) \mathrm{D}(x) \mathrm{D}(y)}{\operatorname{gcd}(\mathrm{D}(x) \mathrm{D}(y), \mathrm{N}(x) \mathrm{D}(y)+\mathrm{N}(y) \mathrm{D}(x))}\right\rfloor, & \text { if } x, y \neq 0 \\ \mathrm{D}(x), & \text { if } y=0 ; \\ \mathrm{D}(y), & \text { if } x=0 .\end{cases}
$$

Note that branching on the 3 cases can be carried out with sign or sgn.

The projection functions are easy to deal with. Now consider how we can push into the operation line $\prod$. Recalling the definition, we have $\mathrm{D}$ (line $\left.\prod_{y=0}^{z} f(y, \bar{x})\right)=\mathrm{D}((1+$ $\left.\lfloor z\rfloor-z) \prod_{y=0}^{\lfloor z\rfloor} f(y, \bar{x})+(z-\lfloor z\rfloor) \prod_{y=0}^{\lceil z\rceil} f(y, \bar{x})\right)$. In $\mathrm{FA}_{\mathbb{Q}}($ disctn), the products up to $\lfloor z\rfloor$ 
and $\lceil z\rceil$ can both be written as legitimate function on their own (this is not the case of $\mathrm{FA}_{\mathbb{Q}}(\mathrm{ctn})$ ). We can push $\mathrm{D}$ into multiplication, addition, and $\lfloor\cdot\rfloor$ (the latter uses reasoning similar to the above functions), so we are just left with the product operation itself. Note that

$$
\mathrm{D}\left(\prod_{y=0}^{\lfloor z\rfloor} f(y, \bar{x})\right)=\frac{\prod_{y=0}^{\lfloor z\rfloor} \mathrm{D}(f(y, \bar{x}))}{\operatorname{gcd}\left(\prod_{y=0}^{\lfloor z\rfloor} \mathrm{D}(f(y, \bar{x})), \prod_{y=0}^{\lfloor z\rfloor} \mathrm{N}(f(y, \bar{x}))\right)} .
$$

The case for summation is similar to products.

The next two propositions are proved inductively on the function algebra; the second one uses the first.

Proposition 6.9 $F A_{\mathbb{Q}}(c t n) \leq \mathcal{T W}$

Proposition 6.10 $F A_{\mathbb{Q}}($ ctn) has a $1 / \mathcal{T} \mathcal{W}$ modulus.

The following corrects lemma 5.18 from [4], making the basic connection between the continuous and discontinuous function algebras.

Lemma 6.11 $F A_{\mathbb{Q}}(c t n) \approx_{+}^{1 / \mathcal{T} \mathcal{W}}\left\{f \in F A_{\mathbb{Q}}(\right.$ disctn $) \mid f$ has $1 / \mathcal{T} \mathcal{W}$ modulus $\}$

\section{Proof}

We prove the two inclusions.

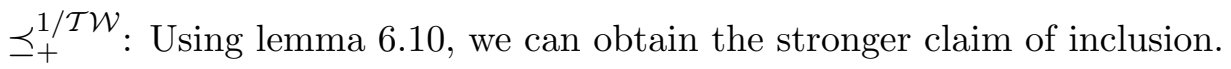

$\succeq_{+}^{1 / \mathcal{T W}}$ : Let $f(x) \in \mathrm{FA}_{\mathbb{Q}}$ (disctn), assuming just one variable for ease of readability.

Furthermore, we assume it has a modulus of $1 / m(x, z)$, where $m(x, z) \in \mathcal{T} \mathcal{W}$. Suppose $\alpha(x, \bar{y}) \in \mathcal{T} \mathcal{W}$, so our goal is now to find some $f^{*}(x, \bar{y}) \in \mathrm{FA}_{\mathbb{Q}}(\mathrm{ctn})$, such that $f \preceq^{1 / \alpha} f^{*}$.

By lemma 6.8, we can assume we have a construction of $f$ in which $\mathrm{D}$ is only applied to the variable $x$. The occurrences of $\mathrm{D}$ in $f$ applied to variables other than $x$ are easily dispensed with since such occurrences of $\mathrm{D}$ must have their variables bound by a sum or product. Sums or products only range over natural numbers, so we can deal with them easily (since for all $u \in \mathbb{N}, \mathrm{D}(u)=1$, except for $\mathrm{D}(0)=0$, so in such cases we can replace $\mathrm{D}(u)$ by a function from $\left.\mathrm{FA}_{\mathbb{Q}}(\operatorname{ctn})\right)$. Thus we can write $f(x)$ as $g(\mathrm{D}(x), x)$, where $g(u, x) \in \mathrm{FA}_{\mathbb{Q}}(\mathrm{ctn})$. Now we give the intuition behind the rest of the proof. We will define $f^{*}(x, \bar{y})$ using $g(u, x)$, so that it approximates $f(x)=g(\mathrm{D}(x), x)$. The difficulty lies in how to get around the discontinuous function $\mathrm{D}$. The idea is to pick a $w \in \mathbb{Q}$ which is sufficiently close to $x$ and such that $\mathrm{D}(w)$ is known in $\mathrm{FA}_{\mathbb{Q}}$ (ctn). By the modulus condition on $f, f(w)=g(\mathrm{D}(w), w)$ will be close to $f(x)$. To carry out the details we will essentially work in $\mathbb{Z}$ and use the linearization to ensure that the function does not fluctuate too much on the rest of $\mathbb{Q}$. We now define some functions in $\mathrm{FA}_{\mathbb{Q}}(\mathrm{ctn})$ (though we will only care about their values on $\mathbb{Z}$ ): 
- $\operatorname{sg}(a)= \begin{cases}1, & a \geq 1 ; \\ -1, & a \leq-1 ; \\ a, & -1<a<1\end{cases}$

- $\operatorname{dv}(a, b)=a * \operatorname{div}(|b|) * \operatorname{sg}(b)(=a / b$ for $a, b \in \mathbb{Z})$

- bottom $(a, b)=b * \operatorname{div}(\operatorname{gcd}(a, b)) * \operatorname{sg}(a)(=\mathrm{D}(a / b)$ for $a, b \in \mathbb{Z})$, recalling that $\operatorname{gcd}$ is always non-negative.

- $h(a, b)=g(\operatorname{bottom}(a, b), \operatorname{dv}(a, b))$

By lemma 4.10, $\hat{h}$ is in $\mathrm{FA}_{\mathbb{Q}}$ (ctn). Recalling the modulus $m$ for $f$, we let $m^{*}(x, \bar{y}) \in$ $F A_{\mathbb{Q}}(\mathrm{ctn})$ be defined so that we have the property

$$
\left|x-\frac{\left[x m^{*}(x, \bar{y})\right]}{\left[m^{*}(x, \bar{y})\right]}\right| \leq \frac{1}{m(x, \alpha(x, \bar{y}))}
$$

By proposition 6.6 we have such an $m^{*} \in \mathcal{T} \mathcal{W}$ that works and by proposition 4.15 we can dominate this $m^{*}$ in $\mathrm{FA}_{\mathbb{Q}}(\operatorname{ctn})$. We define $f^{*}(x, \bar{y})=\hat{h}\left(x m^{*}(x, \bar{y}), m^{*}(x, \bar{y})\right)$. It is in $\mathrm{FA}_{\mathbb{Q}}(\mathrm{ctn})$, and we just need to show that $\left|f(x)-f^{*}(x, \bar{y})\right| \leq 1 / \alpha(x, \bar{y})$. For any $x, \bar{y} \in \mathbb{Q}$, let $(F, x, \bar{y})=$

$$
\begin{aligned}
& \left\{\hat{h}\left(\left\lfloor x m^{*}(x, \bar{y})\right\rfloor,\left\lfloor m^{*}(x, \bar{y})\right\rfloor\right),\right. \\
& \hat{h}\left(\left\lfloor x m^{*}(x, \bar{y})\right\rfloor,\left\lceil m^{*}(x, \bar{y})\right\rceil\right), \\
& \hat{h}\left(\left\lceil x m^{*}(x, \bar{y})\right\rceil,\left\lfloor m^{*}(x, \bar{y})\right\rfloor\right), \\
& \left.\hat{h}\left(\left\lceil x m^{*}(x, \bar{y})\right\rceil,\left\lceil m^{*}(x, \bar{y})\right\rceil\right)\right\} .
\end{aligned}
$$

By proposition 2.9, we know that for any $x, \bar{y} \in \mathbb{Q}: \min (F, x, \bar{y}) \leq f^{*}(x, \bar{y}) \leq \max (F, x, \bar{y})$. Thus it suffices to show that $\left|f(x)-\hat{h}\left(\left[x m^{*}(x, \bar{y})\right],\left[m^{*}(x, \bar{y})\right]\right)\right| \leq 1 / \alpha(x, \bar{y})$. By definition,

$$
\begin{aligned}
\hat{h}\left(\left[x m^{*}(x, \bar{y})\right],\left[m^{*}(x, \bar{y})\right]\right) & =g\left(\mathrm{D}\left(\frac{\left[x m^{*}(x, \bar{y})\right]}{\left[m^{*}(x, \bar{y})\right]}\right), \frac{\left[x m^{*}(x, \bar{y})\right]}{\left[m^{*}(x, \bar{y})\right]}\right) \\
& =f\left(\frac{\left[x m^{*}(x, \bar{y})\right]}{\left[m^{*}(x, \bar{y})\right]}\right)
\end{aligned}
$$

By property and the definition of a modulus, $\left|f(x)-f\left(\frac{\left[x m^{*}(x, \bar{y})\right]}{\left[m^{*}(x, \bar{y})\right]}\right)\right| \leq 1 / \alpha(x, \bar{y})$ and we are done.

Now we will show $\mathcal{L}$ can approximate $\mathrm{FA}_{\mathbb{Q}}(\mathrm{ctn})$.

\section{Lemma 6.12 basic $_{\mathbb{Q}} \preceq_{+}^{1 / \mathcal{T W}} \mathcal{L}$}

\section{Proof}

Except for $\theta_{1}$ and div, $\mathcal{L}$ approximates exactly the functions in basic $\mathbf{C}_{\mathbb{Q}}$ (since it contains extensions of them). For the functions $\theta_{1}$ and div recall lemma 6.4 and note that these two functions are both defined as functions which are a merging of functions approximated in $\mathcal{L}$. For $\theta_{1}$ this is easy. For div we use the fact that $1 / x$ can be 
approximated (for $x \geq 1$ ) because (as observed in [1]) $E(t, x) \in \mathcal{L}$, where $E(t, x)=$ $\left\{\begin{array}{ll}\frac{1-e^{-t x}}{x}, & \text { if } x \neq 0 ; \\ t, & \text { if } x=0 .\end{array}\right.$ To approximate $1 / x$ we then substitute an appropriately fast function for the variable $t$. We then merge this with the constant function 1 at the point $x=1$.

Lemma 6.13 line $\sum$, line $\prod \preceq_{+}^{1 / \mathcal{T W}} \mathcal{L}$

\section{Proof}

First, we claim the following fact:

$$
\text { line } \sum \text {, line } \prod \preceq_{+}^{1 / \mathcal{T} \mathcal{W} \text {,id }\left.\right|_{\mathbb{Z}}} \mathcal{L} \text {. }
$$

In words, the statement means that ignoring their values off of $\mathbb{Z}$, sums and products can be approximated by $\mathcal{L}$. Putting the fact together with lemma 6.5 finishes the proof.

To see why the fact is true, we note that an examination of the proof of lemma 4.7 of [3] essentially proves it (we cannot use their lemma as they state it, because it requires the functions in question to have universe $\mathbb{N}$, but ours can take on vales in $\mathbb{Q}$; ours also have domain $\mathbb{Z}$, rather than $\mathbb{N}$, but this is no problem when working in $\mathcal{L}$ ). For sums, it is immediate. For products, they conclude the accumulated error of their use of $\mathrm{LI}$ is an expression of the form $\epsilon(n) 2^{n}(n+1) \beta^{n+1}(n)$, where $\epsilon(n)=\exp (-\beta(n))$, thus by choosing $\beta(n) \in \mathcal{L}$ growing quickly enough, we can obtain our desired $1 / \mathcal{T} \mathcal{W}$ approximation.

Thus, the next corollary follows by using lemma 3.9 and the above approximations, following the form of the proof in corollary 4.25 (notice that we use lemma 3.13 again, which requires propositions 6.9 and 6.10).

Corollary 6.14 $F A_{\mathbb{Q}}(c t n) \preceq_{+}^{1 / \mathcal{T W}} \mathcal{L}$

\section{Conclusion}

We have introduced two techniques, lifting and approximation, and have applied them to obtain two theorems concerning the elementary functions. In particular, we have extended a result of Bournez and Hainry [1]. We argue that the notion of approximation provides the right connection between function algebras of real functions and computable analysis and could be used to link together different theories of computability over the reals. Another informal claim of this work is that these techniques are general and should be applicable to other complexity classes. This claim is supported by other work in progress (which is perhaps not so convincing to the reader) and by the character of many of the claims which did not depend on the fact that we happened to be working with the elementary computable functions. So of course further work is to apply these techniques more broadly. In particular we have work in progress relating to the class \#P. Furthermore it 
seems that it should be relatively straightforward to apply these techniques to the classes stronger than the elementary computable functions, in particular, to the Grzegorczyk hierarchy up to the primitive recursive functions and recursive functions (such connections have been made in terms of discrete part in [3], and in terms of Computable Analysis in [1] and [2]). More ambitious goals include results of this kind for the weaker complexity classes such as the polynomial time functions.

\section{Acknowledgements}

This work was partially supported by Fundação para a Ciência e a Tecnologia and EU FEDER POCTI/POCI, namely, via CLC, project ConTComp POCTI / MAT / 45978 / 2002, and grant SFRH / BPD / 16936 / 2004.

\section{References}

[1] O. Bournez and E. Hainry. Elementarily computable functions over the real numbers and $\mathbb{R}$-sub-recursive functions. Theoretical Computer Science, 348(2-3):130-147, 2005.

[2] O. Bournez and E. Hainry. Recursive analysis characterized as a class of real recursive functions. to appear, 2006.

[3] M. L Campagnolo, C. Moore, and J. F. Costa. An analog characterization of the Grzegorczyk hierarchy. Journal of Complexity, 18:977-100, 2002.

[4] M. L. Campagnolo and K. Ojakian. The methods of approximation and lifting in real computation. In Third International Conference on Computability and Complexity in Analysis, 2006 .

[5] D. S. Graça. Some recent developments on Shannon's general purpose analog computer. Mathematical Logic Quarterly, 50(4-5):473-485, 2004.

[6] A. Grzegorczyk. Computable functionals. Fund. Math., 42:168-202, 1955.

[7] K.-I. Ko. Complexity Theory of Real Functions. Birkhaüser, 1991.

[8] C. Moore. Recursion theory on the reals and continuous-time computation. Theoretical Computer Science, 162:23-44, 1996.

[9] J. Mycka. $\mu$-recursion and infinite limits. Theoretical Computer Science, 302:123-133, June 2003.

[10] J. Mycka and J. F. Costa. Real recursive functions and their hierarchy. Journal of Complexity, 20(6):835-857, 2004.

[11] D. S. Graça O. Bournez, M. L. Campagnolo and E. Hainry. Polynomial differential equations compute all real computable functions on computable compact intervals. Journal of Complexity, 2007. doi: 10.1016/j.jco.2006.12.005.

[12] K. Weihrauch. Computable Analysis: An Introduction. Springer-Verlag, 2000. 